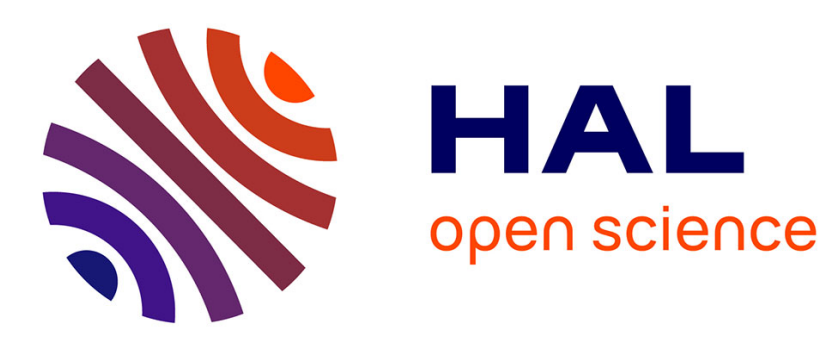

\title{
Nommer/penser sa langue et celle des autres: le cas des grammairiens du sanskrit et des prakrits
}

Emilie Aussant

\section{To cite this version:}

Emilie Aussant. Nommer/penser sa langue et celle des autres: le cas des grammairiens du sanskrit et des prakrits. Histoire Epistémologie Langage, 2009, La nomination des langues dans l'histoire, 31 (2), pp.89-116. halshs-01110680

\section{HAL Id: halshs-01110680 \\ https://shs.hal.science/halshs-01110680}

Submitted on 15 Feb 2015

HAL is a multi-disciplinary open access archive for the deposit and dissemination of scientific research documents, whether they are published or not. The documents may come from teaching and research institutions in France or abroad, or from public or private research centers.
L'archive ouverte pluridisciplinaire HAL, est destinée au dépôt et à la diffusion de documents scientifiques de niveau recherche, publiés ou non, émanant des établissements d'enseignement et de recherche français ou étrangers, des laboratoires publics ou privés. 


\title{
NOMMER/PENSER SA LANGUE ET CELLE DES AUTRES : LE CAS DES GRAMMAIRIENS DU SANSKRIT ET DES PRAKRITS*
}

\author{
Émilie Aussant \\ Laboratoire d'Histoire des Théories Linguistiques \\ Université Paris Diderot - CNRS
}

\begin{abstract}
RÉSUMÉ : L'objet de cet article est de décrire et d'expliquer la manière dont les grammairiens de l'Inde ancienne ( $\mathrm{du} 4^{\mathrm{e}} \mathrm{s}$. avant notre ère jusqu' au $12^{\mathrm{e}} \mathrm{s}$.) nommaient les entités linguistiques. L'étude montre comment ces derniers semblent n'avoir jamais pris en compte l'altérité linguistique externe, pourtant avérée dès le $3^{\mathrm{e}} \mathrm{s}$. avant notre ère. L'altérité linguistique interne, en revanche, n'a pas été ignorée. Mais elle semble - pour la période concernée, tout au moins - avoir toujours été appréhendée sur le même mode, celui du continuum linguistique.
\end{abstract}

MOTS-CLÉS : $\quad$ Inde ; Grammairiens indiens; Altérité linguistique; Continuum linguistique ; Sanskrit ; Variation linguistique ; Prakrit ; apaśabda; apabhramśa ; bhāsā deš́ ;laukika; mleccha; vaidika
ABSTRACT: The aim of this paper is to describe and explain the way in which grammarians of ancient India (from the $4^{\text {th }}$ century B. C. to the $12^{\text {th }}$ century A. D.) named linguistic varieties. The study shows how these scholars seem to have never taken into account external linguistic alterity, though proved from the $3^{\text {rd }}$ century B. C. on. Internal linguistic alterity, on the other hand, was not ignored, but - at least in the period under consideration - it seems to have always been conceived in the same manner, that is, as a linguistic continuum.

KEYWORDS : Indian grammarians ; India ; Linguistic otherness ; Linguistic continuum; Linguistic Variation; Prakrit; Sanskrit ; apaśabda; apabhramśa; bhāsā ; deśi ;laukika ; mleccha; vaidika

\section{INTRODUCTION}

La présente étude vise à examiner et à expliquer la manière dont les entités linguistiques ont été nommées dans l'Inde ancienne. Conformément aux axes de réflexion proposés dans le cadre du projet «La nomination des langues dans l'histoire », je me suis intéressée en priorité à la manière dont une catégorie spécifique d'usagers - les grammairiens - nommait les langues, pensant que cet examen livrerait les clés de l'histoire des représentations de l'objet « langue » dans l'une des plus anciennes traditions grammaticales qui soient. Nous verrons que ces

* Je tiens à remercier, pour leurs remarques et conseils avisés, Nalini Balbir, Christèle Barois, Jean-Luc Chevillard, Pascale Haag, Georges-Jean Pinault, V. Venkataraja Sarma, ainsi que l'ensemble des participants au projet « La nomination des langues dans l'histoire ». 
érudits, tout théoriciens du langage qu'ils étaient, sont loin d'avoir accordé une place centrale à la question de la nomination des langues. Constat bien surprenant à dire vrai, quand l'on songe à l'acuité de ces lettrés et à l'extraordinaire richesse de l'histoire linguistique de l'Inde.

Par «Inde ancienne », j'entends ici une période allant du $4^{\mathrm{e}} \mathrm{s}$. avant notre ère, élaboration de l'Aștâadhyāỹ $(\mathrm{A})$ de Pāninini (acte de naissance de la tradition grammaticale sanskrite pāṇinéenne, la plus ancienne et la plus connue des traditions grammaticales indiennes), jusqu'aux alentours du $12^{\mathrm{e}}$ s., date de composition du Prākrtānuśásana de Purusottama (grammaire prakrite ${ }^{1}$ décrivant plusieurs variétés linguistiques). Cet intervalle chronologique recouvre donc deux moments charnières dans l'histoire linguistique, politique et culturelle de l'Inde : 1) autour du début de notre ère, le sanskrit, jusque là réservé à la classe des clercs, devient progressivement une variété suprarégionale dominant les sphères littéraires, politiques et religieuses ${ }^{2} ; 2$ ) aux alentours de l'an 1000 , les prakrits émergent sur la scène littéraire et politique et entraînent, à partir de là, l'éviction progressive du sanskrit.

Avant d'aborder les principaux textes qui jalonnent ces quinze siècles ${ }^{3}$, je rappellerai brièvement l'histoire de l'indo-âryen (ensemble des parlers d'origine indo-européenne, attestés sur le territoire de l'Inde, tout au long de l'histoire). On distingue généralement trois stades :

1) le stade de l'indo-âryen ancien (sanskrit védique à partir du milieu du $2^{\mathrm{e}}$ millénaire avant notre ère, puis sanskrit classique à partir $\mathrm{du} 4^{\mathrm{e}} \mathrm{s}$. avant notre ère) ;

2) le stade de l'indo-âryen moyen (représenté par le prakrit, qui a évolué en se ramifiant en de nombreuses formes régionales - les prakrits);

3) le stade de l'indo-âryen moderne (langues actuelles de l'Inde du Nord). Une telle présentation laisse penser qu'il y a eu une continuité historique régulière entre sanskrit et prakrit(s) ${ }^{4}$; or il semblerait que ces deux variétés linguistiques aient cohabité depuis une période fort ancienne (on trouve des formes prakrites dans les textes védiques et certains prakrits ont conservé des formes sanskrites archaïques qui n'apparaissent pas dans les textes védiques les plus anciens ${ }^{5}$ ), l'usage du sanskrit étant réservé à l'élite intellectuelle et religieuse (la classe des brahmanes), celui du prakrit aux femmes et autres classes de la société6. Un certain nombre de prakrits sont toutefois restés très proches du sanskrit et laissent penser qu'il était

1 On présente généralement les prakrits comme des dialectes du sanskrit (voir paragraphe suivant).

2 Sans doute sous l'impulsion des conquérants étrangers comme l'Iranien Rudradāman qui l'utilise, vers 150 , dans une inscription. À partir de là, le sanskrit se prête peu à peu à des emplois nouveaux (rédaction du canon dans certaines écoles bouddhiques, de textes scientifiques comme la médecine, etc.).

3 Pour une description succincte des principaux ouvrages cités dans cette étude, se reporter au tableau des repères historiques figurant en fin d'article.

4 De fait, le prakrit est une forme d'indo-âryen plus évoluée que le sanskrit classique.

5 Voir Hock \& Pandharipande (1978, p. 13), Pinault (1989a).

6 C'est dans les œuvres de dramaturgie, où les variétés linguistiques sont distribuées selon le rang social des personnages, que cette situation diglossique se manifeste avec le plus d'évidence. Je reviendrai sur ce point (voir partie $\mathrm{n}^{\circ} 5$ ). 
assez aisé de passer d'une variété à l'autre. Par ailleurs, les locuteurs de ces formes d'indo-âryen étaient en contact avec ceux de langues non indo-européennes, principalement les langues dravidiennes, mais également des langues tribales et, plus ponctuellement, des langues de conquérants étrangers comme le grec $^{7}$. Depuis une date ancienne, donc, le sanskrit a été confronté à une double altérité linguistique, interne et externe ${ }^{8}$.

\section{APERÇU DES DONNÉES VÉDIQUES}

Quand les textes védiques décrivent des luttes entre clans de locuteurs de variétés d'indo-âryen ancien (i.e., sanskrit védique et sanskrit classique ; ces locuteurs se nomment eux-mêmes $\bar{A} r y a$ ), la question de la langue semble - pour ce que jai pu observer - ne jamais être évoquée. Les noms employés ne renvoient apparemment à aucune spécificité linguistique'. C'est lorsqu'il est question d'affrontements opposant ārya et an-ārya (dont certains, parmi ces derniers, parlaient très probablement une langue non indo-européenne ${ }^{10}$ ), qu' apparaissent des expressions faisant référence aux langues. Les an-ārya, principalement désignés sous le nom de dāsa ou dasyu ${ }^{11}$, sont généralement qualifiés au moyen de formules dépréciatives, telles que mleccha, dérivé de la racine mlecch- "parler de manière confuse, indistincte », rendu fréquemment par «barbare $»^{12}$, an-ās (litt. « sans bouche »)

7 Les inscriptions d'Aśoka ( $3^{\mathrm{e}} \mathrm{s}$. avant notre ère) mentionnent les peuples iranien (yavana), grec (yona) et dravidiens (cola, pāndya, keralaputra).

8 Sur l'emploi des expressions «altérité linguistique interne» et «altérité linguistique externe », voir Présentation, partie $n^{\circ} 3.2$.

9 Louis Renou cite, entre autres, les noms de clans suivants : Bharata, Anu, Druhyu, Turvaśa, Yadu, Pūru (1985, § 754). Pour une liste détaillée des noms de clans cités dans les textes védiques, voir Witzel (1999, p. 359). Si la langue qui compose les textes védiques laisse transparaître la coexistence de plusieurs variétés d'indo-âryen, les textes védiques, eux, n'en font pas mention.

10 Plusieurs éléments, dans les textes védiques, indiquent en effet que les Ārya étaient en contact avec des locuteurs de langues diverses ; sur ce point, voir Kuiper (1997, p. 95), Hock (1999, p. 169) et Witzel (1999). Il semblerait que, parmi les an-ārya («non âryens »), aient été classés aussi bien des locuteurs de variétés indo-âryennes que des locuteurs de variétés non indo-âryennes.

11 Manfred Mayrhofer indique, pour dāsa : «m. Dämon, Feind ; Ungläubiger (im Gegensatz zum Ārya), Barbar; Sklave, Knecht [...]. Etymologischer Zusammenhang mit dasyuh (s. uch Dasah) liegt nahe; die ältere Bedeutung wäre dann etwa "aus dem (fremden) Land". » (1963, Band II). Louis Renou note : «Les Dāsa ou Dasyu ont une base ethnique plus certaine, et dans la langue des hymnes le premier de ces mots, qui signifiera plus tard “esclave”, s'oppose à “âryen”. » (1985, § 663).

12 Employé notamment dans un extrait du Śatapathabrāhmana sur lequel je reviendrai dans la partie $\mathrm{n}^{\circ} 3$; il s'agit de la première occurrence du terme. Leemans and Hansman (cités par Driem 2001, p. 1036) proposent une autre analyse de mleccha : il serait lié - comme le pali milakkha - au terme meluhha, qui apparaît dans des documents mésopotamiens en cunéiforme (voir Parpola 1994, p. 13-14, 170), probablement comme ethnonyme harappéen. 
« sans parole $»^{13}$, ou encore krsna-yon $\bar{\imath}$ « d'origine obscure » ${ }^{14}$. La même opposition se retrouve au niveau des glottonymes, où la langue des an-ārya est désignée à l'aide d'expressions telles que mrdhra-vāk «langue inintelligible ${ }^{15}$, asury $\bar{a} v \bar{a} k$ 《langue démoniaque » ${ }^{16}$, et s'oppose à la langue védique, simplement nommée $v \bar{a} k$ (« langue, parole $»)^{17}$ ou devī vak « langue divine », « déesse Parole ${ }^{18}$.

\section{L'ASTTĀDHYĀYI (A) DE PĀNINI}

\subsection{Les faits}

En théorie, les règles générales de la grammaire de Pānini valent pour deux variétés linguistiques ${ }^{19}$ : la variété commune dite bhāsā (litt. «parole », de bhās- « parler, dire ») « langue parlée », qui correspond à la forme classique de la langue sanskrite parlée au nord du sous-continent ${ }^{20}$, et celle dite chandas (litt. « mètre », puis « texte versifié ») « littérature sacrée », qui correspond à une forme ancienne de la langue, conservée dans les textes védiques ${ }^{21}$. Lorsque l'un des deux domaines (car pour

13 Voir Hock (1999, p. 156).

14 Manfred Mayrhofer donne, pour yoni : «m. Weg; Sitz, Aufenthaltsort ; daraus weiterhin, "Mutter-leib, Ursprung, Geburt, Sippe" » (1976, Band III). L'interprétation du mot krṣna («noir/sombre ») reste problématique dans les textes védiques. Certains penchent pour une référence à la race (via la couleur de la peau), d'autres y voient l'association de l'altérité/ adversité à l'« obscurité ». Voir Hock (1999).

15 Manfred Mayrhofer indique, pour mrdhra-vāk: «Schmähreden führend, Übles redend. » (1963, Band II).

16 Manfred Mayrhofer signale, pour asura : «mächtig, m. Herr, später : böser Geist, Dämon. » (1956, Band I).

17 Voir l'hymne à la Parole Rg-veda 10.125 .

18 Voir l'hymne à la Parole $R g$-veda 8.100.11.

19 La première raison qui justifie l'étude de la grammaire de Pāninini, selon Patañjali, c'est qu'elle permet de préserver les textes védiques : seul celui qui connaît les formes de ces textes et les modifications auxquelles elles sont sujettes saura préserver les Veda. Cela présuppose que les faits de langue que décrit l'A sont - notamment - ceux que l'on observe dans les textes védiques. Mais en réalité, l'organisation du système pāninéen se fonde sur la structure du sanskrit classique (la description du système verbal en est une parfaite illustration) et les particularités du védique font l'objet de règles «périphériques », qui dérivent de celles relatives au sanskrit classique. Voir Kiparsky (1979, p. 56-57).

20 On réserve généralement le nom de sanskrit à la forme «classique» de la langue, qui correspond à la variété linguistique décrite par les grammairiens pāninéens, et que les philologues nomment sanskrit classique ou sanskrit littéraire. La forme «ancienne » de la langue, celle des textes védiques, se voit désignée sous le nom de védique. Pānini serait originaire de Śalātura, au Gandhāra. Les faits qu'il décrit relèvent, sauf mention particulière, des variétés linguistiques du nord (voir Thieme, 1935, p. 76 et sq.).

21 Cette variété linguistique est également, bien que plus rarement, désignée sous le terme nigama (litt. «ce par quoi l'on acquiert une connaissance »; voir A 6.3.113, A 6.4.9, A 7.2.64, A 7.3.81, A 7.4.74) et, à l'occasion d'une règle (A 1.1.16), sous celui de ärșa (« relatif à/provenant des rși (« sages ») », i.e., selon la Kāśikāvrtti (KV) «propre aux textes védiques sacrés », c'est-à-dire aux textes védiques récités en continuité (samhitā-pātha), en opposition aux textes védiques non sacrés, c'est-à-dire aux textes védiques récités mot à mot (pada-pātha)). Si le terme chandas désigne, au départ et comme l'indique son nom, les textes védiques versifiés, il finit par renvoyer à l'ensemble des textes védiques ; le critère du sacré l'emporte sur celui du style. Voir Thieme (1935, p. 68-69). 
Pānini, il s'agit bien de domaines d'emploi, comme je le montrerai ci-après) se distingue de l'autre, une règle spécifique est formulée, indiquant le domaine pour lequel elle vaut, comme l'illustrent les deux couples d'exemples suivants :

- Règle générale : A 7.2.87 dvitīyāyām ca

« [La voyelle $\bar{a}$ est le substitut de la finale des thèmes pronominaux yusmad et $\left.a_{s} m a d\right]^{22}$ aussi $(c a)$ devant les désinences du deuxième cas (dvitīyayāạm $)^{23}$. 》

Les formes sont les mêmes, qu'il s'agisse de la langue parlée ou de la littérature védique : $m \bar{a} m$ («me »), tvām («te »), etc.

- Règle spécifique : A 7.2.88 prathamāyāśs ca dvi-vacane bhāṣāyām

« [La voyelle $\bar{a}$ est le substitut de la finale des thèmes pronominaux yusmad et asmad] aussi (ca) devant les désinences duelles (dvi-vacane) du premier cas

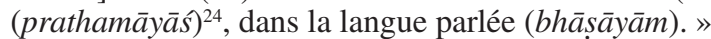

Les formes de la langue parlée sont yuvām et $\bar{a} v \bar{a} m$, distinctes des formes observables dans les textes védiques: yuvam et āvam.

- Règle générale : A 1.4.80 te prāg dhātoh

«Ces [particules gati (type particulier d'unités) et upasarga (préverbes) sont employées] (te) devant (prāg) la racine verbale (dhātoh). »

- Règle spécifique : A 1.4.81 chandasi pare_api

«Dans la littérature sacrée (chandasi), [les particules gati et upasarga sont employées] aussi (api) après [la racine verbale] (pare). »

Les deux domaines de la bhāṣa et du chandas sont eux-mêmes divisés en sous-domaines. Pour le chandas, on en compte près de six. Le principal critère fondant cette division - celui de la stylistique - est clairement indiqué par certains des noms de ces sous-domaines, qui ne sont autres que ceux de différents types de textes qui composent le Veda: certaines formes linguistiques sont propres aux portions en prose, d'autres aux portions versifiées, etc. Les noms des sous-domaines du chandas sont les suivants : 1) mantra (litt. « ce qui sert à penser», dérivé de la racine man- «penser », d'où « formule » $)^{25}$, qui recouvre les sections de textes védiques sous forme de prières versifiées ainsi que des formules sacrificielles en prose et s'oppose à la prose des Brāhmaṇa (voir notamment A $6.1 .151^{26}$, ainsi que

22 La séquence entre crochets est reconduite depuis les règles A 7.2.84 et A 7.2.86.

23 C'est-à-dire l'accusatif.

24 C'est-à-dire le nominatif.

25 Une autre analyse propose la racine $m n \bar{a}$ - (« réciter mentalement, se rappeler») comme étymon de mantra.

26 A 6.1.151 hrasvāe candra-uttara-pade mantre // « Dans un mantra (mantre), [1'accrément suT devant $c$ vaut, dans un composé,] devant le membre ultérieur (uttara-pade) candra, après [un membre antérieur à finale] brève $(h r a s v a \bar{c})$. » La KV donne l'exemple suś-candra et précise qu'en dehors du domaine des mantra, c'est la forme su-candra (sans accrément suṬ) qui prévaut. 
ce qu'en dit Paul Thieme $\left.{ }^{27}\right)$; 2) yajus ${ }^{28}$, qui désigne une formule sacrificielle en prose et qui s'oppose à un vers (voir A 6.1.11729);3) $r c^{30}$, qui désigne une formule versifiée et qui s'oppose à une formule en prose (voir notamment $\left.\mathrm{A} 6.3 .55^{31}\right) ; 4$ ) brāhmana ${ }^{32}$, qui désigne un genre particulier de texte védique (voir notamment A 4.3.105 ${ }^{33}$, ainsi que ce qu'en dit Paul Thieme ${ }^{34}$ ). Le cinquième sous-domaine du chandas se fonde sur un critère pragmatique ; il est désigné sous l'appellation yajña-karmaṇi «dans l'action sacrificielle » (voir A 1.2.34 ${ }^{35}$ ). Quant au sixième sous-domaine, il ne dépend d'aucun des critères cités précédemment; Pānini le désigne à l'aide d'une particule marquant l'option: $v \bar{a}$ «habituellement, préférentiellement» (voir notamment A 1.4.936) ou vibhāṣa $\bar{a}$ «marginalement,

27 1935, p. 38-46.

28 De yaj- «sacrifier, faire don ». Notons que le terme yajus, dans l'A, ne signifie pas « [texte du] Yajur [veda], de même que celui de $r c$ ne signifie pas « [texte du] $\operatorname{Rg}[$ veda] ».

29 A 6.1.117 yajusy urah // « [Le vocatif du mot uru, i.e.] uro (urah) [conserve sa finale en présence d'un $a$ initial également maintenu] dans une formule sacrificielle (yajusy). » On obtient ainsi une séquence telle que uro antarikșam «Ô large ciel! » (au lieu de uro 'ntarikṣam), cas de non-élision observable dans les formules sacrificielles (Thieme, 1935, p. 53).

30 De arc- « louer, honorer ». Le terme $r c$ - signifie « strophe » ou « vers ».

31 A 6.3.55 rcah śe // « Dans une formule versifiée ( $r c a h)$, [la forme pad est le substitut du mot $p \bar{a} d a$ ] devant [le suffixe] śas (śe). » La KV donne l'exemple pacchah (pad + śas) et précise qu'en dehors du domaine des formules védiques versifiées, c'est la forme pādaśah (sans le substitut pad de $p \bar{a} d a$ ) qui prévaut.

32 Litt. «interprétation du brahman », mais aussi «interprétation des brahmanes », « recueil desdites interprétations ». Ces textes consistent essentiellement en observations théologiques relatives aux rites ou aux hymnes et prières. Ils se répartissent selon le Veda dont ils relèvent (il y a les Brāhmana du Rgveda, ceux du Yajurveda, ceux du Sāmaveda et ceux de l'Atharvaveda).

33 A 4.3.105 purāna-proktesu brāhmana-kalpesu // «[Le suffixe secondaire nini vaut au sens de "promulgué par"] quand il s'agit d'un Brāhmaṇa ou d'un texte de rituel (kalpeșu) qui ont été promulgués (proktesu) par d'anciens [maîtres] (purāna). » La KV donne les exemples suivants : bhāllavin « [Brāhmaña] promulgué par Bhallu», paingin « [texte de rituel] promulgué par Pinga» et cite, comme contre-exemple, la séquence yājñavalkāni brāhmaṇāni «Brāhmaṇa promulgués par Yājñavalka », sage considéré comme non ancien.

34 1935, p. 71.

35 A 1.2.34 yajña-karmany ajapa-nyūinkha-sāmasu // «Dans l'action sacrificielle (yajña-karmany), [l'énonciation se fait sur un seul et même ton], excepté quand il s'agit d'une récitation murmurée (ajapa), d'une insertion [de o] (nyūinkha), d'une mélodie (sāmasu). » La KV précise qu'en dehors de l'action sacrificielle, c'est-à-dire dans l'étude (sampāthe), les formules sont récitées avec les accents qui leur sont propres.

36 A 1.4.9 sasthī-yuktaś chandasi vā // «Dans la littérature sacrée (chandasi), [le mot pati "maître" porte] préférentiellement $(v \bar{a})$ [le nom ghi], quand il se construit avec un génitif (sasthi-yuktaś).» Selon que le mot pati porte ou non le nom technique ghi, il suit une certaine flexion: dans une séquence telle que kuluñāanạm pataye namah «Hommage (namah) au maître (pataye) des Kuluñca (kuluñcānām) », la forme pataye (pati comme ghi) est préférentiellement employée à celle de patye (pati comme non ghi). 
rarement » (voir notamment A 6.2.164 $\left.{ }^{37}\right)^{38}$.

En ce qui concerne la $b h \bar{a} s \bar{a}$, les sous-domaines répondent à des critères bien plus variés que ceux du chandas. On peut distinguer : 1) un sous-domaine « régional », que Pāninini désigne soit à l'aide d'un toponyme (tel que prāñc « oriental, à l'Est », voir notamment A $1.1 .75^{39}$, ou encore udañc « septentrional, au Nord », voir notamment A 6.3.32 ${ }^{40}$ ), soit à l'aide d'un ethnonyme (comme sauvīra et sālva, voir notamment A $4.2 .76^{41}$, ou encore uśinara, voir notamment A $\left.2.4 .20^{42}\right)$; 2) un sous-domaine «pragmatique », marqué par des expressions

37 A 6.2.164 vibhāṣā chandasi // «Dans la littérature sacrée (chandasi), [la syllabe finale du membre ultérieur stana d'un composé possessif porte] marginalement (vibhāsāa) [le ton aigu, quand le membre antérieur est un nom de nombre]. » La forme dvi-stana « à deux mamelles », avec ton aigu sur la syllabe initiale, sera employée de préférence à la forme $d v i$ $s t a n \bar{a}$, avec ton aigu sur la syllabe finale.

38 Pour la traduction et la différenciation des termes marquant l'option, voir Kiparsky (1979).

39 A 1.1.75 é̉ prācām deśe // « [Un mot où la première d'entre les voyelles qu'il contient est] $e$ ou $o(e \dot{N})$ [porte le nom technique $v r d d h a$ et peut donc ainsi recevoir le suffixe $c h a$ ] quand il désigne une localité orientale ( $p r a \bar{c} a \bar{m} m$ deśe) / quand il désigne une localité (deśe), selon les maîtres orientaux (prācām). » La KV donne, entre autres, l'exemple d'Enīpacana, nom d'un village oriental qui porte, par conséquent, le nom de $v r d d h a$ et donc auquel le suffixe $c h a$ est applicable, ce qui permet de former enīpacanīya « qui se trouve à Enīpacana ». Par contre, à partir de la forme Devadatta, nom d'un village du Nord-Ouest (qui, par conséquent, ne porte pas le terme technique $v r d d h a$ ), on forme le dérivé daivadatta « qui se trouve à Devadatta » et non daivadattika (formation que le nom de vrddha aurait permis), voir Ojihara et Renou (1967, vol.3, p. 114-115). On notera par ailleurs les deux interprétations de la séquence prācām deśe (les deux étant admises par les commentateurs pāninéens) : la première interprétation, qui considère prācām comme renvoyant à des usages dialectaux, a été très peu défendue par les indologues modernes; la deuxième interprétation, qui considère prācām comme renvoyant à une école orientale de grammairiens, a connu beaucoup plus de succès (sur les deux interprétations et leurs défenseurs, voir Cardona, 1976, p. 147). Les travaux de Madhav M. Deshpande, tenant de la première interprétation, me semblent très convaincants.

40 A 6.3.32 mātara-pitarāv udīcām // « [Le composé copulatif] mātara-pitarau « père et mère » [vaut] chez les septentrionaux (udīcām). » La KV précise qu'ailleurs, c'est la forme $m \bar{a} t \bar{a}-$ pitarau qui prévaut.

41 A 4.2.76 strīṣu sauvīra-sālva-prākșu // «[Le suffixe secondaire a N vaut, par entrave du suffixe $a N$, aux quatre significations susdites, le nom formé étant] féminin (strīsu) [et l'endroit en question étant] chez les Sauvīra (sauvīra), chez les Sālva (sālva), chez les Orientaux (prāksu). » La «signification» du suffixe $a \tilde{N}$ qui vaut dans ce contexte est spécifiée par A 4.2.68 : le suffixe signifie que telle chose a été exécutée par la personne notée par le thème de base en valeur d'instrumental, quand il s'agit d'une localité tirant son nom de cette spécification. Ainsi, chez les Sauvīra, il existe une ville du nom de Dāttāmitrū, nom dérivé de celui du fondateur de la ville, Dattāmitra; chez les Sālva : Vaidhūmāgnī (de Vidhūmāgni); chez les Orientaux : Kākandī (de Kakanda). Dans les régions habitées par d'autres populations, c'est le suffixe $a N$ qui est employé ; la KV donne l'exemple (sous A 4.2.68) de Kauśāmbī nagarī. Les deux types de formes se distinguent seulement par l'accentuation (la première syllabe porte l'accent aigu pour $a \tilde{N}$, le suffixe pour $a N$ ).

42 A 2.4.20 samjñāyām kanthā-uśinnaresu // «[Un composé déterminatif dont le membre ultérieur est] kanthä "cité", quand il s'agit d'un nom propre (samjñāāạm) [désignant une localité chez les] Uśīnara, [est du genre neutre]. » La KV donne l'exemple du nom sauśamikantham, composé du genre neutre désignant une localité chez les Uśīnara, et cite, comme contre-exemple (composé du genre féminin), le nom dākși-kanthā qui désigne une localité qui ne se situe pas chez les Uśīnara. 
telles que ākrośa «insulte» (voir notamment A 3.3.45 $5^{43}$ ), ou encore kutsana « dédain, mépris » (voir notamment A 2.1.5344) ; 3) un sous-domaine «social », indiqué par une expression comme śüdra (voir A 8.2.8345); 4) un sous-domaine « scolastique », que Pānini désigne en citant le nom de théoriciens du langage (tel que Śäkatāyana, voir notamment A 3.4.111 $\left.{ }^{46}\right)^{47}$; 5) comme pour le chandas, un sous-domaine qui n'est conditionné par aucun des critères cités précédemment et que Pāninini désigne à l'aide d'une particule marquant l'option (il y en a trois : $v \bar{a}$ « habituellement, préférentiellement» (voir notamment A 2.3.71), vibhāsa $\bar{a}$ « marginalement, rarement » (voir notamment A 3.1.120) et anyatarasyām « parfois,

43 A 3.3.45 ākrośe_ava-nyor grahah // « [Le suffixe ghañ vaut] après la racine grah- (grahah) précédée des préverbes ava- et ni-(ava-nyor), quand il s'agit d'une insulte (ākrośe). » La KV cite comme exemple la forme nigrāha «malédiction, punition » et, comme contre exemple, la forme nigraha « répression ».

44 A 2.1.53 kutsitāni kutsanaih // «[Les mots désignant] des choses méprisables (kutsitāni) [forment des composés déterminatifs avec un nom fléchi désignant] le mépris [relatif auxdites choses].» La KV cite, comme exemple, le composé vaiyākaraṇa-kha-sūci « grammairien [qui est une] aiguille [à percer] l'espace » autrement dit, un « grammairien stupide » et, comme contre exemple, la séquence kutsito brāhmanah. Dans ce dernier cas, le mot brāhmaṇa devant toujours être employé avec respect, il ne peut y avoir formation d'un composé.

45 A 8.2.83 pratyabhivāde aśüdre // «[La dernière voyelle d'une phrase est protractée et reçoit le ton aigu] quand il s'agit d'une réponse à un salut (pratyabhivāde), excepté [si le salut vient d'un] śüdra (serviteur) (aśüdre). » La KV donne la séquence suivante comme exemple : abhivādaye devadatto_aham - bho āyusmān edhi devadattā3 «Je te salue, moi Devadatta - Sois de longue durée de vie, Devadatta! » et cite, comme contre exemple (le salut vient d'un śüdra), la séquence suivante : abhivādaye tusajako_aham - bho āyusmān edhi tusajaka «Je te salue, moi Tusajaka - Sois de longue durée de vie, Tusajaka!» (la dernière voyelle de la séquence-réponse n'est pas protractée et ne porte pas le ton aigu).

46 A3.4.111 lAN̉ah śākațāyanasya_eva // «Selon Śākatāyana seulement (śākatāayanasya_eva), [Jus est le substitut de jhi de la désinence] d'imparfait (lAN $a h)$ [d'une racine à finale $\bar{a}$ ]. » La KV cite l'exemple ayuh « ils allaient », de la racine $y \bar{a}$-, et donne ayān (même sens) comme contre exemple, i.e., comme forme acceptée par les autres grammairiens.

47 Les autres grammairiens cités par Pānini sont: Āpiśali (A 6.1.92), Bhāradvāja (A 7.2.63), Cākravarmana (A 6.1.130), Gālava (À 6.3.61, A 7.1.74, A 7.3.99, A 8.4.67), Gārgya (A 7.3.99, A 8.3.20, A 8.4.67), Kāśyapa (A 8.4.67), Śākalya (A 1.1.16, A 6.1.127, A 8.3.19, A 8.4.51), Senaka (A. 5.4.112) et Sphotāyana (A 6.1.123). Paul Thieme rappelle à juste titre que, contrairement à l'interprétation consacrée par la tradition pāninéenne, la citation d'un nom de grammairien dans l'A n'indique pas que la règle dans laquelle ledit nom figure est optionnelle; elle signifie que les formes obtenues par la règle sont employées ou enseignées dans les compositions du maître mentionné, en lieu et place de celles prescrites par Pānini (1937, p. 335-337). Il est par ailleurs fort probable que ces formes ne soient pas autre chose que des usages dialectaux, propres à la région du maître cité. 
alternativement » (voir notamment A 1.2.21 $\left.1^{48}\right)^{49}$. On est en droit de s'interroger sur ce dernier genre de variations: l'Aștāadhyāy $\bar{\imath}$ ne les présentant pas comme dépendantes de facteurs morpho-syntaxiques, phonétiques, régionaux, sociaux, stylistiques, pragmatiques ou scolastiques, à quelles conditions sont-elles donc liées ? Comme Paul Kiparsky le souligne, il semblerait que Pāṇini ait simplement souhaité indiquer des préférences linguistiques ${ }^{50}$

\subsection{Analyse}

La situation linguistique décrite par Pānini, nous le constatons, ne fait état d'aucune altérité linguistique externe (aucune mention de langue ou de culture ne faisant pas partie de la zone géographico-socio-religieuse où le sanskrit était parlé, sans doute particulièrement étendue à l'époque de Pānini). Faut-il voir, dans la description des variétés d'usages (les sous-domaines de la bhāsāa), l'indice d'une altérité linguistique interne ? Lorsque Pānini cite le nom d'un grammairien utilisant ou enseignant une forme différente de celle présentée comme générale ou courante dans l'Asțāadhyāy $\bar{l}$, fait-il référence à une forme prakrite ? C'est probable. Ce qui est certain, en revanche, c'est que Pānini ne présente pas ces variations d'usage comme des formes incorrectes et ne les hiérarchise pas : il donne véritablement le sentiment de décrire une seule et même langue qui ne comporte que des variétés liées à des domaines d'emploi. Il n'y a pas, pour lui, de distinction chronologique entre les deux variétés linguistiques que sont la $b h \bar{a} s \bar{a}$ et le chandas, ce dernier ne représente pas pour lui un état plus ancien de la bhāsa $\bar{a}$; il n'y a pas non plus de différence de correction entre une forme employée par tel grammairien ou dans telle région et celle qu'il produirait lui-même. La description pāṇinéenne s'inscrit dans une perspective résolument descriptive et synchronique.

Pānini, tout comme ses premiers commentateurs (voir ci-après), n’emploie pas de nom particulier pour désigner la langue qu'il décrit. Deux raisons, totalement opposées, peuvent être invoquées pour expliquer le fait qu'une langue soit dépourvue de nom «propre »: 1) on ne nomme pas une langue parce que l'on ne reconnaît pas son existence, 2) on ne nomme pas une langue parce que l'on ne reconnaît que son existence (partant, il est évident que l'on fait référence à elle et elle seule). Si Pāninini n'utilise pas de terme particulier pour désigner la langue qu'il décrit, c'est sans doute pour la seconde raison. Cette langue est très vraisemblablement celle

48 A 1.2.21 uD-upadhād bhāva-ädi-karmanor anyatarasyām// [Les suffixes dits niṣthā comportent] alternativement (anyatarasyām) [la susbtitution de guna] après [une racine à] pénultième $u$ ( $u D$-upadha $d$ ), quand il s'agit d'un état, etc. ou d'une action incipiente (bhāva$\bar{a}$ di-karmanor). » Ainsi, il y a option entre les deux formes pradyotitah et pradyutitah « [le soleil] s'est mis à éclairer » (de la racine pra-dyut-).

49 Si l'on s'en tient au nombre de règles, c'est le sous-domaine «optionnel » qui domine (il concerne plus d'une vingtaine de règles, sans compter les reconductions). Vient ensuite le sous-domaine « scolastique » (plus d'une quinzaine de règles), le sous-domaine « régional » (une quinzaine de règles) et les sous-domaines «pragmatique » et «social » (moins d'une dizaine de règles).

50 1979, p. 2-3 
(ou l'une de celles) dont il est locuteur ${ }^{51}$. Cela peut également s'expliquer - même si Pānini ne le formule nulle part dans son traité - par le fait que le sanskrit, dès l'époque védique, n'est pas pensé comme une langue parmi d'autres, mais comme constituant véritablement LE langage : il est unique, éternel (on ne le conçoit pas comme pouvant être créé à aucun point initial du temps) et parfait, puisque d'origine divine ${ }^{52}$. Le sanskrit étant identifié au langage, l'altérité linguistique (externe et interne) n'est pas reconnue, l'individuation de la langue ne s'impose donc pas et, par suite, celle de sa nomination non plus. Mais le fait de décrire cette variété linguistique - et non une autre - est, en soi, révélateur : s'il n'est pas nécessaire de la nommer, puisqu'il ne peut s'agir que d'elle et qu'elle est unique, pour quelle raison Pānini entreprend-il de la décrire ?

L'auteur de l'Astā dhyāȳi appartient à la caste des brahmanes, classe sacerdotale dont l'autorité s'affirme dès les premiers textes indiens qui sont parvenus jusqu'à nous. Sa puissance est essentiellement religieuse, comme le rappelle Madeleine Biardeau :

Elle est aussi la caste qui manie le sanskrit, langue des textes sacrés, et elle est seule habilitée à l'enseignement religieux. Elle est gardienne de la culture brahmanique d'une façon générale, des cultes et pratiques orthodoxes et, partant, de l'ordre social. ${ }^{53}$

L'époque de Pānini est marquée par des transformations profondes dans le domaine des croyances religieuses : les institutions mises en place par les Ārya sont concurrencées par des courants émergeants, tels le bouddhisme et le jaïnisme, qui aspirent à la délivrance du cycle des renaissances et remettent ainsi en cause certains des fondements de la société brahmanique. Ces courants mettent également en question la suprématie de la langue sanskrite, cette dernière n'étant pas la langue de prêche de leur fondateur (le Bouddha aurait recouru à diverses variétés linguistiques, Mahāvīra - fondateur du jaïnisme - aurait employé le prakrit ardhamāgadhī). Cette situation peut donc aussi, d'une certaine manière, expliquer la non nomination de la langue sanskrite : si, comme le rappelle Cécile Canut : « [...] le nom n'existe que pour s'opposer à d'autres noms, c'est à l'autre qu'il s'adresse, c'est par rapport à l'autre qu'il se définit $\aleph^{54}$, nommer la langue sanskrite, c'est aussi reconnaître l'existence des autres langues, en particulier celles qui véhiculent les doctrines bouddhiste et jaïne.

Je terminerai ce chapitre par quelques remarques relatives à la diversité des facteurs dont dépendent les variations décrites par Pāninini. Concernant le chandas,

51 Un examen rapide de la grammaire de Pānini montre qu'il ne s'agit pas d'un manuel pratique du sanskrit : sa composition (en sanskrit), son organisation et son fonctionnement ne répondent pas aux besoins d'un débutant dans cette langue, c'est une grammaire qui s'adresse à des locuteurs avertis. Par ailleurs, Pānini mentionne un certain nombre de tournures familières (voir Renou, 1956, p. 63-64, 67 et 82-83) qui laissent penser que cette langue n'était pas restreinte à un usage religieux et qu'elle était pratiquée - par ceux qui y étaient autorisés - dans un contexte informel.

52 La parole, émanation du divin, est déifiée dès le Rgveda. Quant à son éternité, elle est étroitement liée à celle des Veda : pour les grammairiens - comme pour d'autres théoriciens du langage - l'éternité des Veda vient de celle des mots qui les composent.

53 1964, p. 13.

54 2001, p. 445 
nous avons pu noter que les sous-domaines étaient principalement désignés par des noms de textes ou de types de textes. La raison en est la suivante : la variété linguistique dont Pānini est locuteur est moins ancienne que celle des textes védiques ; pour décrire les faits propres au chandas, il n'a d'autre choix que de se référer auxdits textes. Pour ce qui est de la $b h \bar{a} s \bar{a}$, je l'ai évoqué à plusieurs reprises, Pānini décrit la (ou l'une des) langue(s) qu'il parle, langue qui ne dépend pas des règles qu'il formule. Cette situation lui permet d'appréhender les diverses variations d'usage sans y apporter de considérations morales et de leur accorder une place au sein de son traité.

\section{LES VĀRTTIKA (V) DE KĀTYĀYANA ET LE MAHĀBHĀṢYA (M) DE PATAÑJALI}

\subsection{Les faits}

Tout comme Pānini, Kātyāyana et Patañjali ne recourent à aucun nom particulier pour désigner la langue qu'ils décrivent. Kātyāyana emploie, en plus de quelques-uns

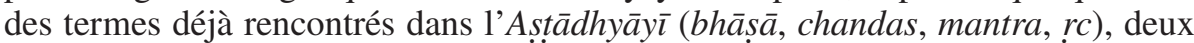
« nouvelles » unités : veda ou vaidika «védique » et loka «monde » ou laukika «mondain », pour désigner respectivement le domaine du védique et celui de l'usage courant ${ }^{55}$, ainsi que les formes qui y sont employées. Avec Patañjali, le champ des expressions servant à qualifier les formes linguistiques s'enrichit et révèle une conception bien différente de celle de Pānini. À la division bhāsāalchandas et à la sous-division de ces deux domaines, opérées de manière totalement neutre, succède une attitude normative opposant, de manière partiale, ce qui est « correct »

55 Ces deux termes correspondent en partie au chandas et à la bhạsā de Pāṇini, termes que Kātyāyana emploie également. Notons que si les termes chandas et bhāsa sont strictement assignés à la désignation de formes linguistiques, aussi bien dans l'A que dans ses commentaires, les mots veda/vaidika et lokallaukika, à partir de Kātyāyana, renvoient également à des pratiques (langagières ou non) qui se distinguent de celles observables dans la métalangue (désignée par les termes tels que vyākarana « analyse » ou «formation diversifiée [des mots] » (voir Pinault, 1989b, p. 319-320), śāstra («traité »), etc.). Ainsi, sous la règle A 1.1.1 vrddhir ādaic, Kātyāyana explique (V 4 et 5) que l'on reconnaît un terme technique grammatical à partir de l'usage qu'en font les grammairiens (domaine de la métalangue), tout comme on reconnaît le nom propre d'un individu à partir de l'usage qu'en font les proches dudit individu (domaine de l'usage courant) et tout comme on reconnaît le nom d'un objet du rituel à partir de l'usage qu'en font les prêtres (domaine védique). 
à ce qui est « corrompu $~^{56}$. Ainsi, Patañjali oppose les formes employées dans les domaines d'emploi dits veda/vaidika et loka/laukika aux formes qu'il qualifie d'apa-śabda «forme corrompue » (le préfixe apa- marque l'absence ou, comme ici, l'infériorité), d'apa-bhramśa «forme déviante » (de bramsś- « dévier ») ou encore, en une occasion, de mieccha $($ śabda $)$ « barbarisme $»^{57}$.

Le tout premier passage du Mahābhāsya (M) dans lequel figurent quelques-unes de ces formes est une citation du Śatapathabrāhmaṇ ${ }^{58}$, un texte védique qui se situerait aux alentours des $8^{\mathrm{e}}-7^{\mathrm{e}} \mathrm{s}$. avant notre ère. L'extrait condamne une conduite (le parler corrompu lors de l'acte rituel) par le biais d'un récit à l'issue négative (la défaite des démons) :

te_asurā helayo helaya iti kurvantah parābabhūvuh / tasmād brāhmanena na mlecchitavai na apabhāsitavai / mleccho ha vā esa yad apaśabdah / mlecchā mā bhüma ity adhyeyạ̣ vyākaraṇam / (M, vol. 1, p. 2)

Ces démons, ayant dit helayo helayah («eh ennemis, eh ennemis »), furent vaincus ; c'est pourquoi le brahmane ne doit pas dire de barbarisme (mlecchitavai) [c'est-à-dire,] ne doit pas parler de manière corrompue (apabhāsitavai), car il est bien connu que le mot corrompu (apaśabdah) est un barbarisme (mleccho). Ne devenons pas des barbares (mlecchā), étudions la grammaire.

Cet extrait s'inscrit dans la section de l'introduction du Mahābhāsya consacrée aux buts de l'enseignement grammatical. L'idée évoquée ici est la suivante: celui qui aura reçu un enseignement grammatical accomplira le rituel en parlant correctement, donc ne se comportera pas comme un barbare et, par conséquent, ne sera pas «vaincu». Kaiyata (auteur $-11^{\mathrm{e}} \mathrm{s}$. ? - du plus ancien commentaire complet du Mahābhāssya) nous apprend que plusieurs éléments peuvent être considérés comme « corrompus » dans la séquence helayo helayah prononcée par

56 Dans le passage qui suit, cependant, des variantes régionales sont mentionnées sans être jaugées : tad yathā / savatir gati-karmā kambojesv eva bhāsito bhavati vikāra enam āryā bhāṣante śava iti / hammatih surāsțtreșu ramhatih prācya-madhyeșu gamim eva tv äryāh prayuñjate / dātir lavana-arthe prācyesu dātram udīcyesu // (M, vol. 1, p. 9-10) «Par exemple, śavati est employé dans le sens d' «aller » chez les Kamboja seulement ; les Ārya l'emploient au sens de «décomposition» : śava (« cadavre »); [dans le sens d'《aller »], les Surāsțra emploient hammati, les [peuples du] moyen Orient emploient ramhati, mais les Ārya emploient seulement gami. Dans le sens de « couteau », les [peuples de] l'Orient emploient dāti, ceux du Nord, dātra. » Dans cet extrait, nous pouvons relever, en plus d'āryā, des termes d'ordre purement géographique (prācya-madhya «moyen Orient», prācya «Orient », udīcya « Nord» ainsi que des ethnonymes formés sur des noms de contrées (kamboja, nom d'une région du Pamir ; surāṣtra, nom d'une contrée de l'Ouest (actuelle Surat)). Madhav M. Deshpande, dans son article de 1978 (p. 94), note qu'une séquence fort semblable se trouve déjà dans le Nirukta (Nir) de Yāska (Nir 2.2).

57 J'ai relevé seulement trois occurrences de cette forme, apparaissant toutes dans un passage qui n'a pas été composé par Patañjali puisqu'il s'agit de la citation d'un texte védique (voir partie $\mathrm{n}^{\circ} 1$ et passage du Satapathabrāhmana cité ci-après).

58 Śatapathabrāhmana (3.2.1.23-24, p. 63) : te_asurā àtta-vacaso he alavo he alava iti vadantah parābabīūvuh / tatra etām api vācam üduh upāijijñāsyām sa mlecchas tasmān na brāhmano mlecchet $[. .$.$] / « Ces démons, parce que privés de langage [correct], ont dit$ he_alavo he_alavah ("eh ennemis, eh ennemis») [et] furent vaincus. Et cette parole énigmatique qu'ils prononcèrent est un barbarisme, c'est pourquoi un brahmane ne doit pas dire de barbarisme [...]. » Pour d'autres occurrences de ce passage, voir Limaye (1974, p. 6). 
les démons ${ }^{59}$ : selon certains, he doit avoir une voyelle protractée (d'une durée de trois mores) et le $a$ initial de alayah ne doit pas être amuï; selon d'autres, seul le mot alayah doit être redoublé (non la phrase helayah) et c'est la forme arayah qui est attendue, non alaya $h^{60}$. Cette citation (qui se termine en glose de ladite citation) du Śatapathabrāhmana présente un double intérêt : 1) sur le plan de la terminologie, elle montre qu'entre le texte de départ (voir note 58) et la glose qui en est donnée dans le Mahābhāṣa, deux «nouvelles » expressions ont fait leur apparition ${ }^{61}$, à savoir apaśabda « forme corrompue » et apabhās- « parler de manière corrompue », formes à forte connotation dépréciative ; 2) le second intérêt de cette citation tient à son contexte initial : le Śatapathabrāhmana fait partie de ces textes védiques - les Brāhmaṇa - qui décrivent les rites : l'homme à qui l'on enjoint de ne pas prononcer de formes corrompues au moment du rituel est un brahmane, un «prêtre », membre de la caste sacerdotale, la plus élevée de la hiérarchie. Autrement dit, ce premier extrait montre clairement comment l'appréhension de formes linguistiques non sanskrites (qu'il s'agisse de formes sanskrites « corrompues » ou de formes étrangères) s'enracine, dès le départ, dans des considérations de nature socio-religieuse.

La section de l'introduction du Mahābhāsya traitant de la méthode d'enseignement contient un autre passage intéressant :

bhūyāmso_apaśabdā alpīyāmsạ̣ śabdāhl eka-ekasya hi śabdasya bahavo_apabhramśăh / tad yathä/ gaur ity asya śabdasya gāvi goñ̄ gotā gopotalikā_ity evam àdayo_apabhramiśạ̣ / (M, vol. 1, p. 2)

Les formes corrompues (apaśabdā) sont les plus nombreuses, les mots [corrects] sont les plus rares. En effet, pour chaque mot [correct,] il y a de nombreuses formes déviantes (apabhramśāh ). Par exemple, pour le mot [correct] gauh (« bovin »), il y a les formes déviantes (apabhraṃśăh) gāvi, goṇi, gotā, gopotalikā, etc.

Outre la forme apabhramśa « forme déviante » qui, comme apaśabda et apabhās-, met clairement en évidence la vision dualiste de Patañjali, deux éléments retiennent notre attention dans cet extrait : 1) les formes qui illustrent la notion d'apabhramśa, à savoir gā $\bar{\imath}$, gon $\bar{\imath}$, gotā et gopotalikā, sont très probablement des formes dialectales d'indo-âryen ${ }^{62}$, ce qui indiquerait que le Mahābhāṣya a été composé dans un contexte d'altérité linguistique interne ; 2) ces formes, présentées comme corrompues, sont en surnombre par rapport aux formes correctes sanskrites.

Un dernier passage de l'introduction du Mahābhāsya doit être signalé :

59 La forme helayah (helayo si le mot suivant commence par une consonne sonore ou un a), avant application des opérations phonétiques, est he alayah.

60 tatra ke cid āhuh - hai-he-prayoge hai-hayoh [A 8.2.85] iti plute prakrti-bhāve ca kartavye tad-akaranam mlecchanam iti / pada-dvir-vacane vākya-dvir-vacanam latvam ca mlecchanam ity apare / (Pradīpa (P), vol. 1, p. 23). Bhartṛhari, dans sa Mahābhāssyadì pikā (MD), mentionne d'autres «fautes », en plus de celles-ci (voir MD, 1962, p. 9). Selon Paul Thieme, ces formes appartiennent à un vernaculaire âryen de l'Est, la māgadhī (1955, p. 437 et 439). Sur la distribution de $r$ et de $l$, voir Hock and Pandharipande (1978, p. 20).

61 Ces deux formes ne semblent pas avoir été employées dans les textes védiques.

62 Voir Pischel (1900, p. 274), Bubeník (1998, p. 27) et Witzel (1999, p. 341). Patañjali cite d'autres formes « corrompues » : devadinna (pour devadatta, voir M sous V 2 ad A r rlK, vol. 1, p. 20), ānapayati (pour ājñāpayati), vattati (pour vartate), vaddhati (pour vardhate), kas (pour krs-), dis (pour drś-) (voir M sous V 12-13 ad A 1.3.1, vol. i, p. 259). 
evam hi śrūyate / yarvānas tarvāno nāma-rșayo babhūvuh pratyaksa-dharmānah

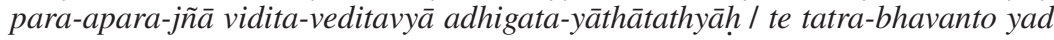
vā nas tad vā na iti prayoktavye yarvānas tarvāna iti prayuñjate yājñe punah karmaṇi na_apabhāṣante / (M, vol. 1, p. 11)

Ainsi, on raconte que des sages du nom de Yarvāna et Tarvāna percevaient la nature des choses, connaissaient l'ici-bas et l'au-delà, savaient ce qui peut être su [et] avaient atteint la réalité. Ces honorables sages, là où il fallait prononcer yad vā nah et tad vā nah, disaient yarvānah et tarvānah ; mais au cours de l'action sacrificielle, ils ne parlaient pas de manière corrompue (apabhāsante).

Le fait notable de ce passage, déjà relevé par Madhav M. Deshpande ${ }^{63}$, tient en ce que même des sages (rṣi), en l'occurrence des personnes accomplissant des actes rituels (yājñe karmani), emploient, dans l'usage courant, des formes dites corrompues. Dans le même ordre d'idée, on peut citer le bref extrait suivant du Mahābhāsya :

aśaktyā kayā cid brāhmanyā ṛtaka iti prayoktavya ltaka iti prayuktam / (M sous V 1 ad $r ! K$, vol. 1, p. 19)

Du fait de son incapacité, une brahmane prononce ltaka là où l'on doit prononcer rtaka.

L'emploi de /l/ en lieu et place de /r/, qui serait caractéristique des variantes linguistiques de l'Est, est présenté - depuis la période védique - comme l'exemple typique de corruption ${ }^{64}$. Notons par ailleurs qu'il est question d'une femme brahmane ; cette précision est loin d'être anodine : elle est l'indice d'une discrimination (linguistique) sexiste, dont on trouve des traces dans d'autres contextes, comme le théâtre (voir note 94).

Ces deux derniers passages montrent donc que, à l'époque de Patañjali, la prononciation erronée des formes sanskrites s'observe jusqu'au sein des classes les plus élevées.

\subsection{Analyse}

Les éléments relevés dans les extraits présentés ci-dessus témoignent d'un changement certain dans la manière de nommer les formes linguistiques en présence, et donc, dans la manière de les appréhender : à la description neutre de Pānini succède une attitude normative, ouvertement fondée sur des considérations socio-religieuses, qui oppose les formes correctes - les seules qui puissent générer un mérite religieux - aux nombreuses formes corrompues. Un autre fait doit être mentionné : à partir de Kātyāyana - soit dès le $3^{\mathrm{e}} \mathrm{s}$. avant notre ère - les expressions pāṇinéennes du type prācām (voir note 39) ou śākatāyanasya (voir note 46), qui servaient à marquer, de manière fort subtile, les conditions d'une variation, ne sont plus considérées que comme un moyen parmi d'autres d'indiquer une option générale.

Comment expliquer cette attitude normative et hiératique ? Deux raisons - une raison « de surface » et une raison « profonde »- peuvent être évoquées. Tous les enseignements pāninéens - qu'ils soient généraux ou liés à un sous-domaine -, et eux seuls, sont appréhendés, par les successeurs (même les plus immédiats)

63 1978, p. 98.

64 Voir Hock et Pandharipande (1978, p. 15). 
du grammairien, comme produisant des formes sanskrites « correctes ». Cette nouvelle façon d'appréhender l'enseignement pāninéen a deux effets: 1) une forme qui ne suit pas les règles de l'Aștāadhyāy $\bar{l}$ est nécessairement considérée comme déviante (ce qui conduit à un effet « 2 bis » : l'élévation du traité, au fil des siècles, au rang de norme ${ }^{65}$ ) ; 2) puisque seule compte la correction, peu importe les conditions des variations, tant qu'elles sont décrites par Pānini ${ }^{66}$. Voilà pour la raison «de surface». Maintenant, comment expliquer ce changement dans le rapport à l'enseignement grammatical, i.e., comment expliquer qu'après Pānini, seuls les enseignements de ce dernier soient considérés comme produisant des formes correctes ? Il semblerait que la période au cours de laquelle Patañjali a composé le Mahābhāṣya ait été marquée par une forte rivalité entre le sanskrit et les prakrits, ces derniers étant même employés, en contexte informel (donc très probablement comme langues maternelles), par les élites (voir le passage relatif aux deux sages Yarvāna et Tarvāna). Le tout premier extrait est, à cet égard, particulièrement éloquent : si les démons ont été vaincus, c'est parce qu'ils parlaient de manière corrompue également lors de l'accomplissement des actes rituels. Autrement dit, l'emploi d'une forme sanskrite corrompue semble être toléré pour l'usage courant, non pour le contexte rituel; autrement dit encore, l'usage de la langue sanskrite, évincée de la communication courante par diverses formes de moyen indien, se limite aux contextes académique et rituel ${ }^{67}$. La communauté brahmanique, maîtresse du culte, apparaît comme la seule à préserver la connaissance et la pratique (comme langue seconde) de la langue sacrée. Elle est en contact quotidien avec des communautés exclues des pratiques rituelles et ne parlant pas sanskrit. Dans ce contexte, l'enseignement grammatical revêt une dimension toute singulière.

Un autre point doit également être souligné. Même s'il oppose l'emploi du sanskrit correct, parlé par les brahmanes, aux formes déviantes énoncées par les non (ou les « mauvais-es ») brahmanes, Patañjali ne dit pas que les formes corrompues ne permettent pas de transmettre le même message que les formes correctes. Formes correctes et formes déviantes sont égales sur ce point. Nous remarquons, en effet, que dans la plupart des passages cités, la corruption se limite à la prononciation ; la formation des mots et la syntaxe semblent encore épargnées.

Je terminerai sur deux éléments de première importance pour notre réflexion. Les termes employés par Kātyāyana et Patañjali renvoient exclusivement à des domaines

65 Norme éternelle qui plus est, puisque valant pour les textes védiques, eux-mêmes éternels.

66 L'aplanissement dans la description des variations peut également trouver son origine dans l'idée - déjà évoquée - selon laquelle la langue sanskrite est éternelle, idée sans doute bien plus prégnante à l'époque de Patañjali qu'à celle de Pānini (sur ce point, voir Deshpande, 1993). L'idée d'une langue éternelle, sans début ni fin et, donc sans évolution, s'accorde difficilement avec celle de variation.

67 Ce changement de situation se perçoit également au niveau des fonctions que les théoriciens du langage prêtent à la grammaire. Certains faits mentionnés par Pānini laissent penser que ce dernier décrivait une variété linguistique vivante (i.e., parlée dans un contexte informel) dans le but de présenter son fonctionnement ; pour Patañjali - qui le formule expressément dans l'introduction du $\mathrm{M}$-, la grammaire vise avant tout à préserver les Veda. En forçant un peu le trait, on peut dire que l'on passe d'une approche où la langue est décrite pour elle-même à une approche où la langue est décrite pour lire et mémoriser les textes qu'elle compose. 
d'emploi et/ou aux formes qui sont employées dans ces domaines. Les expressions apaśabda « forme corrompue », apabhramśa « forme déviante », mleccha(śabda) «barbarisme », etc. ne désignent que des mots, elles ne sont jamais employées pour renvoyer à une langue. En outre, le fait d'appréhender ces formes comme des corruptions, autrement dit, comme des formes sanskrites mal prononcées ou mal formées, montre assez clairement que, dans l'esprit de ces grammairiens, l'on est en présence d'un continuum linguistique ${ }^{68}$ : il n'y a qu' un langage (le sanskrit, védique ou classique) et des variations, plus ou moins corrompues, de ce langage. Cette appréhension non-différenciatrice du paysage linguistique était déjà présente dans l'approche « variationniste » et synchronique de Pānini, mais elle était alors vierge de tout jugement de valeur. Avec Kātyāyana et Patañjali, la perspective variationniste se double d'une dimension « déviationniste».

\section{LE VĀKYAPADİYA DE BHARTṚHARI}

\subsection{Les faits}

Le Vākyapadīya de Bhartrihari contient un passage particulièrement intéressant. Voici les stances qui le composent ${ }^{69}$ :

śabdah sạ̣skāra-hīno yo gaur iti prayuyuksyate I

tam apabhramśam icchanti visisista-artha-niveśinam // 1.175

Le mot (śabdah) dépourvu d'apprêt [grammatical] (samskāra-hīno) que l'on souhaite employer [en tant que mot] gauh (« bovin »), est, par rapport à cet objet particulier (i.e., le bovin), une forme déviante (apabhramśam).

Vrtti

śabda-prakrtir apabhramśa iti samgraha-kārah / [...] tatra gaur iti prayoktavye_aśakty $\bar{a}$ pramāda-ādibhir vā gāvy-ādayas tatprakrtayo_apabhramśāh prayujyante I

«Une forme déviante a pour fondement un mot [correct] », dit l'auteur du Samgraha ${ }^{70}$. [...] Là où on devrait employer gauh («bovin »), par incapacité ou par négligence, etc., on emploie des formes déviantes (apabhraṃsāha) telles que $g \bar{a} v \bar{l}$, etc. qui ont [la forme gauh] pour fondement.

asva-gony-ādayaḥ śabdāh sādhavo viṣaya-antare /

nimitta-bhedāt sarvatra sādhutvam ca vyavasthitam // 1.176

Les mots tels que asva, goṇ̄, etc. sont corrects (sādhavo) quand ils [sont employés] pour [désigner] un autre objet [que les mots aśva et go]. Et on détermine toujours la correction [d'un mot] à partir de ce qui le différencie [dans sa] cause [d'application].

te sādhuṣv anumānena pratyaya-utpatti-hetavah /

68 Voir Aklujkar (1996, p. 74-75).

69 Certaines sont présentées avec des extraits de leur glose (Vrtti).

70 Comme le remarque Koduvayura Anantarama Subramanya Iyer dans son article de 1964, si cette phrase a bel et bien été prononcée par l'auteur du Saṃgraha - mentionné à partir de Kātyāyana, cet ouvrage n'est pas parvenu jusqu'à nous -, à savoir Vyādii, cela signifie que le mot apabhraṃśa était employé avant Kātyāyana et Patañjali. 
tādātmyam upagamya_iva śabda-arthasya prakāśakāh // 1.177

Ces [formes asva, gon̄ī, etc.] causent la naissance de la compréhension, par inférence, des [formes] correctes (sādhușv); comme en tendant à l'identité [avec ces formes correctes], elles mettent en lumière l'objet du mot.

Vrtti

[...] atha kasmād ete go-śabdasya gāvy-ädayah paryāyā na vijñāyante / na hi śișta-samācāra-prasiddher anyad evam prakāreșu smrti-nibandhaneșv artheșu nimittam abhidhīyate / gāovy-ādayaś cet paryāyāh syur ete _api śistair laksanair anugamyeran prayujyeramśs cal yaś ca pratyaksa-paksena prayojakeșv abhidheyesu pravartate sa sādhuh / sākșāt tu prayojakam vācyam artha-rūpam sādhubhih pratyāyyate / tasmād à ha-

[...] Maintenant, pourquoi ne reconnaît-on pas ces formes gāvī, etc. comme synonymes du mot go ? En effet, quand il s'agit de choses de ce genre, qui sont fondées sur la tradition, rien d'autre que ce qui est connu par l'usage des gens bien éduqués n'est considéré comme une cause. Si les formes telles que $g \bar{a} v \bar{l}$, etc. étaient des synonymes [de la forme gauh], les gens bien éduqués les décriraient à l'aide de règles et les emploieraient. Et [un mot] qui dénote directement l'objet qui cause son emploi, c'est [un mot] correct; et l'objet à exprimer qui cause l'emploi [du mot] est connu directement à l'aide des [mots] corrects. C'est pourquoi [Bhartrhari] dit :

na śistair anugamyante paryāyā iva sādhavah /

te yatah smrti-śāstrena tasmāt sākṣād avācakāh // 1.178

Les gens bien éduqués, parce qu'ils [se fondent] sur le traité de la tradition (i.e., la grammaire), n'acceptent pas [ces formes erronées] comme des synonymes corrects ; pour cette raison, elles ne font pas comprendre [leur objet] directement.

ambv-aṃbv iti yathā bālah śikșamāno_apabhāṣate I

avyaktạ̣ tad-vidām tena vyaktau bhavati niścayah // 1.179

De même que [lorsqu'] un enfant, apprenant [à parler], dit de manière corrompue (apabhāsate) ambu ambu ${ }^{71}$, ceux qui savent [la forme correcte en retirent] la connaissance sûre [de la forme énoncée de manière] distincte ;

evam sädhau prayoktavye yo_apabhramśah prayujyate I

tena sādhu-vyavahitah kaś cid artho_abhidhīyate // 1.180

de même, la forme déviante (apabhramśah) qui est employée là où il faut employer une forme correcte ( $s \bar{a} d h a u$ ), désigne un objet par l'intermédiaire de [la forme] correcte.

Vrtti

sañkīrnāyām vāci sādhu-vișaye_apaśabdāh prayujyante / taih śiștā lakṣaṇavidah sādhün pratipadyante / tair eva sādhubhis tad artham abhidhīyamānam paśyanti / anumānas tu dhüma iva_agner asādhur itareșām //

Dans la langue mêlée (sañkīrnāyām vāci), les formes déviantes (apaśabdāh) sont employées à la place des [mots] corrects $(s \bar{a} d h u)$. À partir de ces [formes déviantes], les gens bien éduqués qui connaissent les règles [grammaticales] rétablissent les [formes] correctes. C'est par ces seules [formes] correctes qu'ils voient l'objet exprimé ; il s'agit-là d'une inférence : c'est [la forme] incorrecte

71 Le terme ambu peut être compris de deux manières, comme le note Jan E. M. Houben dans son article de 1996 (p. 182, note 29) : il peut s'agir d'une prononciation incorrecte de $a m b \bar{a}$ «mère » (amba au vocatif) ou de ambu « eau » (ambo au vocatif). 
[qui fait inférer] le reste, comme la fumée [fait inférer] le feu.

pāramparyād apabhramiśā viguṇeșv abhidhātrṣu /

prasiddhim āgatā yena teșām sādhur avācakah // 1.181

Pour ceux qui parlent mal (viguṇeșv abhidhātrșu), ce sont les formes déviantes (apabhramśa $)$ qui, par la force de la tradition, sont bien connues ; de fait, une forme correcte (sādhur) ne signifie rien pour eux.

daivī vāg vyatikīrneyam aśaktair abhidhātrbhih /

anitya-darśinām tv asmin vāde buddhi-viparyayah // 1.182

Cette divine parole (daivī $v \bar{a} g)^{72}$ est déréglée par ceux qui la parlent sans en avoir la capacité (aśaktair abhidhātrbhih) ; mais chez les tenants de la non-éternité [des mots], c'est l'opinion contraire [qui prévaut] concernant cette doctrine ${ }^{73}$.

\subsection{Analyse}

Sur le plan strictement terminologique, nous retrouvons la vision dualiste du Mahābhāṣya, peut-être légèrement plus marquée : les « formes correctes » $(s \bar{a} d h u)$, sont opposées aux «mots dépourvus d'apprêt grammatical » (śabdah samskārahìnah $)^{74}$ qui sont énoncés par « ceux qui parlent mal » (viguneṣv abhidhätrșu) ou « ceux qui parlent [sanskrit] sans en avoir la capacité » (aśaktair abhidhātrbhih).

Sur le plan du contenu des stances, trois éléments retiennent particulièrement notre attention. Il y a, en premier lieu, ce parallèle établi entre les mots incorrects que prononce un enfant et les formes déviantes de ceux qui «parlent mal du fait de leur incapacité » (stances 1.179-1.180). Ce que suggère ce rapprochement, c'est que, tout comme l'enfant parle - de manière incorrecte - la même langue que ses parents, les locuteurs qui produisent des formes déviantes parlent la même langue

72 À propos de l'usage de daivī vāc chez Bhartrhari, Ashok Aklujkar écrit ce qui suit (1996, p. 73-74) : «Bhartrhhari speaks of daivi vāc in a context in which it must refer to the language approved by the Pāninian grammarians - to what we call Sanskrit, as well as to the language that existed in an age free from contamination and corruption - in a golden age. Thus, a continuity is implied between Sanskrit, on the one hand, and the Veda language and/or the Language Principle, on the other, and the adjective "divine" is used in such a way as to connote its applicability throughout. »

73 Dans son article de 1996 (p. 184-188), Jan E. M. Houben montre comment la première partie de cette stance expose, dans la continuité des stances 1.175 à 1.181 , la vue des grammairiens sanskrits et comment la seconde partie évoque une vue opposée, peut-être jaïne ou bouddhiste (ou, selon Kahrs 1992, p. 242, logicienne). Cette seconde vue rejette, entre autres, la déification du sanskrit.

74 C'est-à-dire aux mots dont la formation n'est pas décrite dans le traité pāṇinéen. Le terme samskāra ne manque pas de rappeler celui de samskṛta, nom qui finira par désigner la langue décrite et codifiée par Pāninini. Dans le contexte brahmanique, la première occurrence de samskrta - en tant qu'adjectif qualifiant ladite langue - se trouve dans le Rāmāyaṇa, l'un des monuments de la littérature indienne, dont la composition pourrait être située - avec la plus grande prudence - entre le IV et le $\mathrm{I}^{\mathrm{er}} \mathrm{s}$. av. J.C. Cette occurrence oppose la samskrtā vāk (« langue/parole apprêtée »), langue des « deux-fois-nés » (dvi-ja; membres des castes supérieures ayant reçu l'initiation), à la mānuṣam vākyam «phrase/parole humaine », qualifiée d'arthavat «pourvue de sens » (entendons : seulement pourvue de sens, contrairement à la saṃskrtā vāk, qui est pourvue de sens et d' " apprêt » grammatical, ce qui signifie qu'elle a été purifiée par l'analyse grammaticale pāninéenne). Sur ce passage, voir notamment Aklujkar, 1996, p. 70-71 et Pollock, 2007, p. 44-45. Cette dimension de la parole purifiée par l'apprêt grammatical est très présente chez Bharțhari. 
que ceux qui n'en produisent pas. On retrouve ici l'idée de continuum linguistique : les formes perçues comme déviantes ne sont pas interprétées comme l'indice d'existence d'une autre langue, mais comme des transgressions de LA langue/ du Langage. Dans le même ordre d'idée, nous notons que le terme apabhramísa renvoie, ici aussi, à un type de mots et non à une langue ${ }^{75}$. Le second élément qui nous intéresse concerne la manière dont l'écart entre les formes correctes et les formes incorrectes est appréhendé. Alors que Patañjali ne remettait pas en cause le fonctionnement linguistique des formes dites corrompues - celles-ci faisant comprendre leur sens selon le même processus cognitif que les formes correctes -, Bhartrhari évoque une vue - qu'il considérerait comme acceptable ${ }^{76}$ selon laquelle, les formes incorrectes, pour les locuteurs du «bon » sanskrit, ne fonctionnent pas de la même manière que les formes correctes. Les stances 1.176 à 1.180 sont très explicites : les formes gâa $\bar{l}$, gonī, etc., corruptions de la forme sanskrite gauh, ne sont pas des synonymes de cette dernière car elles font connaître leur objet de manière indirecte, via une inférence de la forme gauh, et c'est véritablement cette dernière, inférée, qui fait comprendre l'objet <bovin>. Enfin, les stances 1.179 à 1.181 révèlent, de manière imagée (1.179-1.180) ou non (1.181), une dissymétrie au niveau des locuteurs : ceux qui parlent correctement, à l'image des adultes qui maîtrisent la langue, comprennent les formes correctes et, quoique de façon indirecte, les formes incorrectes ; ceux qui parlent de manière corrompue, en revanche, ne comprennent que les formes incorrectes.

Ce durcissement de la vision normative que donne à voir le Vākyapadīya s'expliquerait par une évolution sensible de la situation du sanskrit. Aux alentours de l'ère chrétienne, le sanskrit serait définitivement passé - pour ses locuteurs, les membres de l'élite - au statut de langue seconde, fruit d'une éducation spécifique. Dans les siècles qui suivent, la grammaire devient une source nécessaire à l'apprentissage de la langue et l'enseignement des trois grammairiens (Pāṇini, Kātyāyana et Patañjali, qui forment le munitraya « la triade de sages ») est élevé, par la tradition des lettrés indiens, au rang de norme et de référence du bon langage ${ }^{77}$. À partir du moment où les grammairiens n'ont plus d'accès direct à l'usage du sanskrit, que leurs seules sources sont les travaux de leurs prédécesseurs, ces derniers acquièrent une autorité qui s'établit de fait. Ce n'est qu'à partir de cette époque que

75 On notera toutefois que ce continuum linguistique commence à se lézarder : la stance 1.181 semble reconnaître que les formes déviantes, pour ceux qui ne connaissent qu'elles, sont «bien connues », donc bien établies, comme si elles constituaient un système autonome, fonctionnant en marge de celui du sanskrit. La question de l'intercompréhension apparaît, en filigrane.

76 Voir Houben (1996, p. 188).

77 Bhartrhhari est le premier grammairien à qualifier Pāṇini, Kātyāyana et Patañjali de śișta ou de rssi. C'est donc à partir du moment où l'accès au sanskrit n'est plus direct (les seules sources sont les travaux des grammairiens antérieurs) que la «trinité de sages » revêt une dimension quasi-religieuse. Voir Deshpande (1998). 
la langue a été appelée, dans les outils linguistiques, samskrta «sanskrit $»^{78}$. La tradition des lettrés donne le mot samskrta comme signifiant la forme de parole « construite » ou « apprêtée » par la grammaire de Pānini, c'est-à-dire lorsqu'elle est accompagnée de la conscience de ses structures de formation. Par sa description, Pāninini réalise véritablement un « acte de vérité » (satya-kriyā) : il révèle au locuteur l'organisation de la langue. Et un locuteur pourvu d'une conscience linguistique aiguë peut empêcher l'évolution spontanée de sa langue. La grammaire de Pāninini dote donc ses utilisateurs d'outils qui leur permettent de ne pas s'écarter du bon usage de leur langue et donc, de le conserver.

\section{LE PRĀKRTĀNUŚĀSANA DE PURUSOTTAMA}

La plus ancienne grammaire prakrite $\left(3^{\mathrm{e}}-5^{\mathrm{e}} \mathrm{s}\right.$. ?) qui soit parvenue jusqu'à nous est le Prākrtaprakāśa de Vararuci. Cet ouvrage, composé en sanskrit, décrit «les règles phonétiques et morphologiques de formation des mots appartenant au dialecte prakrit de base, la māhārāsțtī littéraire, à partir de la norme sanskrite (formulation de type «au lieu de... on a...»). $»^{79}$ Il s'adresse à un public qui connaīt le sanskrit et qui souhaite composer de la poésie en māhārāsțrī. Le trait le plus frappant de l'œuvre de Vararuci, pour ce qui nous intéresse ici, est l'absence presque totale de glottonymes. Si l'on fait exception du titre (Präkrtaprakāśa $)^{80}$, qui nous offre l'une des premières occurrences du terme prākrta (litt. «situé dans la prakrti » ou « qui vient de la prakrti », la prakrti («base ») étant le sanskrit ${ }^{81}$ ) dans le contexte grammatical, la langue décrite elle-même reste innommée ; le seul autre nom propre de langue employé est celui de samskrta (substantif), à la fin de l'ouvrage ${ }^{82}$. En revanche, et c'est là encore bien plus surprenant, l'un des héritiers directs de Vararuci, Puruṣottama (fin $11^{\mathrm{e}}$, début $12^{\mathrm{e}} \mathrm{s}$.), fait usage de près de trente-deux termes (dont vingt-deux noms propres) pour désigner des variétés linguistiques.

\subsection{Les faits}

Le grammairien bouddhiste Purusottama serait le plus ancien représentant de l'école dite « orientale » de grammaire prakrite ${ }^{83}$. Son ouvrage, le Prākrtānuśāsana, composé en sanskrit, décrit les règles phonétiques et morphologiques permettant de former les mots de la māhārāsțīi littéraire à partir de la norme sanskrite, ainsi que les règles propres aux variétés linguistiques s'écartant de la norme prakrite

78 C'est également à partir de cette période que les domaines d'emploi de la langue ainsi nommée se multiplient: inscriptions, oeuvres séculières, exégèse et commentaires des textes bouddhiques et jaïns sont progressivement rédigés en sanskrit, langue transrégionale, transethnique et achronique, qui finit par dominer (jusqu'au début du deuxième millénaire) les sphères politique, littéraire et religieuse. Voir Hock et Pandharipande (1978, p. 18-19) et Pollock (2007).

79 Balbir (2000a, p. 267).

80 Luigia Nitti-Dolci cite les autres titres qui ont été attribués à l'ouvrage (1938, p. 13-14).

81 Voir Kahrs (1992, p. 229-245).

82 śeșah saṃskrtāt // (9.18) « Le reste [est à inférer] à partir du sanskrit. »

83 Voir Nitti-Dolci (1938, p. 89) et Balbir (2000b, p. 273). Selon Bubeník (1998, p. 44 et 47), il aurait été précédé par Kramadīśvara, auteur du Sạ̣kșiptasāra. 
établie.

L'intérêt de cette œuvre réside dans le classement - sans précédent dans les grammaires - de variétés en quatre catégories : bhạsa $\bar{a}, v i b h \bar{a} s \bar{a}^{84}$, apabhramśs $a^{85}$ et paiśācika ${ }^{86}$. Parmi les bhāṣă, la māhārāștrī littéraire (ainsi nommée dans l'œuvre de Purusottama) occupe une place de choix ; son étude - qui s'appuie toujours sur celle de Vararuci - sert de base à la description des autres variétés. Les quatre autres bhāṣa sont : la śaurasen̄̄, la prācyā, l'āvantī et la māgadhī. La catégorie des vibhāṣā regroupe quatre variétés : la śākarīin, la cāndalī, la śābarī et la takkadeśî ; celle des apabhramśa en compte huit : le nāgaraka, le vrācadaka, l'upanāgaraka, le pāñcala, la vaidarbhī, la lātî̀, la kaikeyī et la gaudīi ${ }^{88}$. La catégorie paiśācika se compose de trois variétés de paiśācī (litt. « langue des démons ») : la variété kaikeya, la variété śaurasena et la variété pāñcāla. En dehors de cette classification, on note l'emploi du terme deśi ( « régionalisme $»^{89}$ ), celui de samskrta (pour désigner le sanskrit) ${ }^{90}$ et celui de prākrta .

En réalité, cette classification de variétés linguistiques en quatre catégories n'est pas sans précédent. Elle s'inspire très certainement de celle que l'on trouve dans le Nātyaśāstra (NŚ), « Traité de dramaturgie » attribué à un certain Bharata, qui daterait des alentours du début de notre ère ${ }^{92}$. Cet ouvrage, qui constitue aussi la plus ancienne étude consacrée à la poétique sanskrite ${ }^{93}$, comporte un chapitre

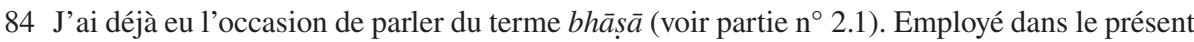
contexte, c'est-à-dire en opposition à vibhāsăa, il revêt le sens de « langue [principale] », i.e., langue fréquemment employée dans les oeuvres littéraires ; vibhạsāa (litt. « langue déviante », le préfixe $v i$ - marquant le manque ou la corruption) désigne les « langues secondaires », i.e., peu fréquemment employées dans les oeuvres littéraires. George A. Grierson rappelle par ailleurs que vibhās $\bar{a}$, chez les grammairiens sanskrits, désigne un type d'option : « We have seen that, as a technical term of grammar, it meant "option", and this, to a certain extent, indicates the meaning of the word as applied to dialects. A vibhāsā is an optional form of a standard dialect used for certain purposes. » (1918, p. 515).

85 Nous avons vu (voir partie $\left.n^{\circ} 3.1\right)$ que le terme apabhramśa signifiait littéralement « forme déviante ». Nalini Balbir rappelle $(2000$ b, p. 274) : « [...] en contexte moyen-indien, terme désignant les prakrits tardifs à l'origine des parlers néo-indiens ».

86 Nalini Balbir précise (2000b, p. 274): «[...] litt. "langue des démons"; forme de moyen-indien attestée de manière très sporadique ». D'aucuns y ont vu le nom d'une langue dravidienne, parlée par une ethnie tribale ; d'autres, comme Oskar von Hinüber, ont montré qu'il s'agissait d'une variété orientale de moyen indien bouddhique (voir Hinüber, 1985).

87 Également appelée śakāra-vacana (13.14) ou śakāra-bhāṣā (13.15).

88 Il est également fait mention, dans cette catégorie des apabhramśa, du parler des Dhakka, de celui des Vakkara (?), de celui des Kuntala, de celui des Pāndi et de celui des Simghala (18.23).

89 Dérivé de deśa (m.) « endroit, région, localité, pays ». Le mot deśī est notamment employé dans le chapitre consacré aux règles phonétiques de la māhārāștrī, à propos du mot thera: therādayo deśyāh // (4.36) « thera, etc. sont des régionalismes. »

90 Employé notamment à la fin de la section consacrée à la māhārāșțīi : śeṣam saṃskrtāt // (8.12) « Le reste [est à inférer] à partir du sanskrit. ».

91 Employé notamment dans la section consacrée à la variété takkadeśī : hariścandras tv imām takka-bhāsām apabhramśam icchati na prākrtam // (16.10) « Mais Haricandra veut que cette langue takka (takka-bhāṣām) soit un apabhramśa et non un prakrit. »

92 Voir Bubeník (1998, p. 33). L'établissement du texte du Nātyaśāstra pose de nombreux problèmes ; la question de la datation ne peut être abordée qu'avec une extrême prudence.

93 Voir Kane (1961, p. 10). 
entier dédié aux variétés linguistiques que l'on peut utiliser sur scène, selon les personnages ${ }^{94}$, ainsi qu'un fragment de grammaire prakrite rédigé en prakrit (fait exceptionnel dans la mesure où la métalangue de la grammaire prakrite est le sanskrit) ${ }^{95}$. Le tableau linguistique que dresse ce traité révèle un foisonnement dialectal d'une incroyable richesse, sans doute très proche de ce qu'était la situation - plurilingue - de l'époque ${ }^{96}$, même si d'aucuns aiment à répéter que les variétés représentées sur la scène ne sont en rien celles de la réalitée ${ }^{97}$. Ce traité distingue d'abord entre samskrta ${ }^{98}$ et prākrta ${ }^{99}$, puis entre $b h \bar{a} s \bar{a}^{100}$ et vibhāsa $\bar{a}^{101}$. On note également l'emploi de deśī « régionalisme » (NŚ 17.3), deśa-bhāsă (litt. « langue régionale») «dialecte»(NŚ 17.24, 17.46) et sva-deśa-jāa (bhāas $\bar{a})$ «[langue]

94 Le sanskrit est propre aux brahmanes et aux rois ; il peut être assigné - selon le temps et la situation, précise Bharata (NŚ 17.38) - à la reine principale et aux filles de ministres, aux nonnes bouddhiques et aux courtisanes, mais la langue des héroïnes et de leurs amies est la śaurasenī (NŚ 17.51). Les autres formes de prakrit sont réservées aux personnages de rang inférieur (serviteurs, bouffons, soldats, etc.). Cette répartition des variétés linguistiques - qui révèle une situation de diglossie - n'est pas sans rappeler l'usage de la «langue vulgaire » dans l'ancien théâtre européen, langue réservée à certains personnages, dont l'emploi - facultatif - ne répondait pas à un souci pédagogique (les personnages qui usent de la langue vulgaire emploient aussi la variété haute). Sur les langues, codes et conventions de l'ancien théâtre européen, voir Bordier (2002).

95 Le prakrit décrit n'est pas nommé de manière spécifique. Selon Manmohan Ghosh, il s'agit probablement de la śaurasen̄i (1934, p. 24). Il est moins probable qu'il s'agisse de la māhārāsțīī, nom absent des listes données dans le Nātyaśāstra.

96 Notons que le théâtre est décrit comme une imitation des conduites du monde, il représente la nature humaine dans sa diversité.

97 Voir notamment Bansat-Boudon (1992, p. 161 et suiv.). L'auteure rappelle (p. 157 et 165) que les prakrits du théâtre figurent parmi les «conventions scéniques qui ne sont pas de ce monde-ci » (alaukikī nātya-dharm $\bar{\imath})$ et non parmi les « conventions qui sont de ce mondeci » (loka-dharmī). Abhinavagupta, auteur du seul commentaire du Nātyaśāstra qui nous soit parvenu - l'Abhinavabhāratī, $\mathrm{XI}^{\mathrm{e}} \mathrm{s}$. -, qualifie en effet la diversité des langues d' « extraordinaire » (alaukika-bhāsāa-ädi-bheda) (voir Bansat-Boudon, 1992, p. 65).

98 Bharata semble être le premier auteur à employer le substantif samskrta en tant que nom de langue.

99 C'est également dans le Nātyaśāstra que l'on trouve la plus ancienne occurrence d'une tripartition des mots prakrits (samāna-śabda, vibhrașta, deśí-gata) ; la classification en tatsama, tad-bhava et deśī remonterait à Dandin (VII s. ?). Sur ce point, voir Kahrs (1992, p. 227).

100 La catégorie des bhāṣā comporte sept variétés: māgadhī, āvantī, prācyā, śaurasen̄̄, ardhamāgadhī, bāhlīkā et dākșiṇātyā. Quatre de ces noms se retrouvent dans la classification de Purusottama.

101 La catégorie des vibhāsāa se compose de sept variétés également : langue des Śakāra (langue des Śabara, dans l'édition de Bombay 1894, p. 183), langue des Ābhīra, langue des Candāla, langue des Śabara (langue des Śacara, dans l'édition de Bombay 1894, p. 183), langue des Dramila (langue des Dravida, dans l'édition de Bombay 1894, p. 183), langue des Andhra (langue des Udra, dans l'édition de Bombay 1894, p. 183) et variété linguistique inférieure des habitants de la forêt (hīnā vane-carānām $)$. Trois de ces sept noms se retrouvent dans la classification de Purusottama. Une remarque sur Dramila/Draviḍa : ce terme d'origine tamoule - dont on tirera dravidien, voir Ghosh (1934, p. 14) - désignerait, non pas une langue dravidienne, mais la variété indo-âryenne parlée en terre dravidienne, par des migrants indoâryens ; voir Grierson, (1918 p. 512-513 et 516). De même, le terme Udra désignerait la variété indo-âryenne parlée en Orissa par des migrants indo-âryens (voir Grierson, 1918 p. 509 et 516). 
venant de sa propre région », mleccha-śabda «barbarisme » (NŚ 17.28), ainsi que d'expressions telles que $e-k \bar{a} r a-b a h u l a \bar{a} b h a ̄ s a \bar{a}$ «langue où le son /e/ abonde » (NŚ $17.57)^{102}$. L'ouvrage réfère parfois à lui-même en employant le terme veda, bien que la langue dans laquelle il a été composé ne possède aucun des traits de la langue védique ${ }^{103}$.

\subsection{Analyse}

Trois innovations terminologiques méritent toute notre attention: 1) le couple samskrta-prākrta, 2) les noms propres de langues tels que māhārāsțtrī, māgadhī, etc., 3) les termes deśí ou deśa-śabdaldeśa-bhāsā.

J'ai déjà eu l'occasion d'évoquer le terme samskrta ${ }^{104}$. Le nom prākrta, je le rappelle, signifie « qui vient de la prakrti » ou « qui est situé dans la prakrti », la prakrti («base ») étant le sanskrit ${ }^{105}$. La première interprétation ( « qui vient de la prakrti ») sous-entend que les prakrits sont des variétés dérivées du sanskrit ${ }^{106}$. La seconde (« qui est situé dans la prakrti ») ${ }^{107}$ laisse penser que ces variétés font partie intégrante d'un univers ou ensemble linguistique (la prakrti), et qu'elles constituent des alternatives, des options, auxquelles il est possible de recourir dans certains contextes $^{108}$. De ce point de vue, les formes prakrites sont appréhendées comme des substituts des formes sanskrites, les deux types d'éléments coexistant au sein d'un même continuum linguistique ${ }^{109}$. Un certain nombre de faits tendent à aller dans le sens de la seconde interprétation, parmi lesquels : 1) la structure générale des règles des grammaires prakrites (« au lieu de $x$ (forme sanskrite) on a $y$ (forme prakrite) ») ; 2) le fait que la grammaire prakrite soit, dans la plupart des cas, conçue comme un appendice à la grammaire sanskrite ${ }^{110}$. Quelle que soit l'interprétation retenue - bien que cela apparaisse de façon bien plus nette dans la seconde -, il semble que nous soyons toujours en présence d'une conception « continuiste » des variétés linguistiques. L'autre terme employé pour désigner les prakrits principaux, à savoir $b h \bar{a} s \bar{a}$, est tout particulièrement remarquable. Recourir au terme même que Pānini employait pour désigner sa propre langue n'est certainement pas anodin ; accéder au rang de bhāsă dénote une certaine importance. Le Nătyaśăstra de Bharata,

102 Les autres expressions sont: na-kāra-bahulām bhāsām «langue où le son /na/ abonde » (NŚ 17.58), ca-kāra-bahulām bhạșām « langue où le son /ca/ abonde » (NŚ 17.59), u-kārabahulām bhạṣām « langue où le son /u/ abonde » (NŚ 17.60) et o-kāra-bahulām bhāṣām « langue où le son /o/ abonde » (NŚ 17.61).

103 Voir Ghosh (1934, p. 12).

104 Voir partie $n^{\circ} 4.2$.

105 Voir Kahrs (1992, p. 228-229).

106Eivind Kahrs (1992, p. 240) rappelle qu'il s'agit d'une dérivation « intellectuelle » (i.e., processus de formation d'un mot dans l'esprit du grammairien), non d'une dérivation historique (ce qui impliquerait l'idée d'un changement dans le temps).

107 Cette interprétation a été proposée par certains grammairiens indiens. Elle a par ailleurs été évoquée par Grierson (1901, p. 553 note 2) et Kahrs (1992).

108 Selon la première interprétation, l'analyse équivaut à dire «telle forme sanskrite devient telle forme prakrite»; selon la seconde, l'analyse revient à dire : «dans tel contexte, telle forme prakrite se substitue à telle forme sanskrite ». Voir Kahrs (1992, p. 234)

109 Voir Kahrs (1992, p. 245).

110 Voir Nitti-Dolci (1938, p. 7). 
qui établit une classification des variétés selon leur emploi sur la scène, montre clairement que certains prakrits avaient toute leur place dans la littérature sanskrite dramatique, même s'ils ne bénéficiaient pas du même prestige que le sanskrit. Je prends soin de préciser «certains prakrits », car la terminologie indique que les autres variétés (vibhāsāa, apabhramía, paiśăcika) restent associées à une certaine « infériorité ». La hiérarchie, finalement, n’a fait que se déplacer, imposée par les pratiques linguistiques des brahmanes : à partir du moment où ces derniers n'ont plus eu, comme langue première, que des variétés prakritiques, leur attitude s'est modifiée, les poussant à considérer sous un jour nouveau (et bien plus favorable) ce qu'ils appréhendaient, quelques siècles plus tôt, comme n'étant que corruption ou déviation. D'où l'emploi de termes tels que prākrta ou bhāsā pour désigner ce qui était qualifié au départ d'apaśabda ou d'apabhramśa. L'intérêt porté aux prakrits s'assume véritablement avec la composition des premières grammaires prakrites autour du $10^{\mathrm{e}} \mathrm{s}$., bien après le Nätyaśāastra.

La plupart des noms propres de langues sont dérivés de noms de régions, telles la māhārāsțrī « [langue de la région] du Mahārāșțra », la māgadhī " [langue de la région] du Magadha », l'āvantī « [langue de la région] de l'Avantī », la prācyā « [langue] de l'Est »; d'autres, moins nombreux, sont dérivés de noms d'ethnies, comme la śaurasen̄̄ «[langue] des Śurasena (ethnie de Mathurā)». Pourtant, dans le Nätyaśāstra, le premier ouvrage - qui nous soit parvenu - à employer ces glottonymes, le critère qui fonde la répartition des langues est avant tout social : le statut et la profession des personnages sont bien plus invoqués que la terre d'origine. Louis Renou - reprenant Bloch 1934, p. 10 - émet l'idée d'un lien avec les confréries d'acteurs :

Sans être inventés, à coup sûr, ces dialectes ont subi une grande stylisation ; il faut y chercher des documents moins sur les parlers réels que sur l'origine des confréries d'acteurs et de bardes (Bloch) : ainsi, la śaurasenī serait le parler des troupes qui à partir de Mathurā ont répandu le théâtre à travers l'Inde. ${ }^{111}$

Si la terminologie invite à penser que l'on est en présence d'une première classification (et donc à un premier stade d'individualisation) de variétés linguistiques, le but qui préside à l'entreprise des grammairiens prakrits (et, avant eux, des théoriciens de dramaturgie) - à savoir composer correctement dans chacune des variétés - semble révéler que ces « parlers » sont plus appréhendés comme des registres ou des genres littéraires que comme des langues à part entière :

Un auteur devait trouver exposés là [i.e., dans les «grammaires » du prakrit] les préceptes nécessaires pour écrire des gāthā en mahārāștrīi, [...] des rôles en dialecte pour un drame du type de Mrcchakatika [...], des vers ou des poèmes en apabhraṃśa comme le Prākrtapingalasūtra [...], et des contes sur le modèle de la Brhatkathā. ${ }^{112}$

Un mot, enfin, sur les expressions deśī « régionalisme » (voir note 89), deśaśabdaldeśa-bhāṣa «mot/langue régional(e)», dont les occurrences, dans le Nātyaśāastra, seraient parmi les plus anciennes (voir Pollock 2007, p. 93). L'emploi du mot deśa ( « endroit, région, pays, royaume ») pour désigner des mots, mais aussi et surtout, des langues, introduit une brèche dans la translocalité-transethnicité du

111 Renou (1985, § 111).

112 Nitti-Dolci (1938, p. 127). 
continuum linguistique - et, in fine, dans le continuum lui-même - : si les variétés linguistiques nommées māhārāstrī, māgadhī, etc. ne sont que des niveaux de langue, des styles ou des genres, a-régionaux, a-culturels et a-chroniques, les deśa-bhāṣa « langues régionales » se définissent avant tout par leur dépendance à une région donnée. Cet ancrage régional (ou cette «vernacularisation », pour reprendre les mots de Pollock) mettra un terme, à partir de la seconde moitié du $20^{\mathrm{e}} \mathrm{s}$., à la conception continuiste des variétés linguistiques et rendra possible l'émergence des langues.

\section{CONCLUSION}

Je terminerai en mettant l'accent sur deux points.

1) Si l'on fait exception de la période védique, l'altérité linguistique externe semble n'avoir jamais été prise en compte par les grammairiens indiens dont les travaux ont été évoqués dans cette étude. C'était pourtant une réalité, dont on trouve trace, dès le $\mathrm{III}^{\mathrm{e}}$ siècle avant notre ère (voir note 7 ).

2) L'altérité linguistique interne, en revanche, n'a pas été ignorée. Mais elle semble - pour la période qui nous concernait, tout au moins - avoir toujours été appréhendée sur le même mode, celui du continuum linguistique : le variationisme non hiérarchisé de Pānini, le variationisme «déviationiste » de Patañjali et Bhartṛhari ${ }^{113}$, le variationisme «débridé »-mais néanmoins hiérarchisé - de Purusottama sont autant de déclinaisons de la même intellection. Il n’y a, finalement, qu'un langage (le sanskrit décrit par Pānini) et des variations (registres, genres, niveaux...), ordonnées ou non, de ce langage.

\section{REPÈRES HISTORIQUES ET ABRÉVIATIONS DES TITRES DE TEXTES INDIENS}

\begin{tabular}{|c|c|c|}
\hline Auteur & Date & Titre de l'ouvrage et genre \\
\hline Pāṇini & $\begin{array}{l}4^{\mathrm{e}} \text { s. av. } \\
\text { J.C. ? }\end{array}$ & $\begin{array}{l}\text { Aștādhyāy } \bar{\imath}(\mathrm{A}) \text { «(Traité) en huit leçons » } \\
\text { Traité grammatical permettant la génération des formes correctes } \\
\text { du sanskrit, fondateur de l'école pạninéenne, rédigé en sūtra ou } \\
\text { «aphorismes » }\end{array}$ \\
\hline Yāska & $\begin{array}{l}7^{\mathrm{e}}-3^{\mathrm{e}} \text { s. av. } \\
\text { J.C. ? }\end{array}$ & $\begin{array}{l}\text { Nirukta (Nir) «Explication » } \\
\text { Libre commentaire des Nighaṇtu du Rgveda, consistant en } \\
\text { interprétations et étymologies de mots védiques }\end{array}$ \\
\hline Kātyāyana & $\begin{array}{l}3^{\mathrm{e}} \text { s. av. } \\
\text { J.C. }\end{array}$ & $\begin{array}{l}\text { Vārttika (V) «Éléments d'interprétation » } \\
\text { Commentaire d'une partie des sūtra de l'Aștādhyāy } \bar{\imath}\end{array}$ \\
\hline Patañjali & $\begin{array}{l}2^{\mathrm{e}} \mathrm{s} . \mathrm{av} . \\
\text { J.C. }\end{array}$ & $\begin{array}{l}\text { Mahābhāsya (M) «Grand commentaire » } \\
\text { Commentaire des Vārttika }\end{array}$ \\
\hline
\end{tabular}

113 Les grammairiens pāninéens ultérieurs appréhenderont les faits de la même manière (voir notamment la Vaiyākaraṇasiddhāntaparamalaghumañjūṣā de Nāgeśa p. 45-48 - dans l'édition de Baroda, 1961 : on retrouve les termes apabhramśa, apaśabda, asādhu, mleccha). 


\begin{tabular}{|c|c|c|}
\hline Bharata & $2^{\mathrm{e}} \mathrm{s} . ?$ & $\begin{array}{l}\text { Nātyaśāstra (NŚ) «Traité de dramaturgie » } \\
\text { Traité encyclopédique relatif au Nātya, englobant la danse, la } \\
\text { musique, le chant, les techniques proprement théâtrales et la } \\
\text { poétique }\end{array}$ \\
\hline Vararuci & $3^{\mathrm{e}}-5^{\mathrm{e}}$ s. ? & $\begin{array}{l}\text { Prākrtaprakāśa «Éclaircissement du prakrit » } \\
\text { Ensemble des règles phonétiques et morphologiques permettant } \\
\text { de former les mots de la māhārāsțtrī à partir de la norme sanskrite }\end{array}$ \\
\hline Bharț̣hari & $5^{\mathrm{e}} \mathrm{s}$ & $\begin{array}{l}\text { Vākyapadīya «(Traité de) la phrase et du mot » } \\
\text { Traité de philosophie grammaticale } \\
\text { Mahābhāsyadīpika (MD) « Lumière sur le grand commentaire » } \\
\text { Commentaire incomplet du Mahābhāssya }\end{array}$ \\
\hline $\begin{array}{l}\text { Vāmana } \\
\text { - Jayāditya }\end{array}$ & $7^{\mathrm{e}} \mathrm{s}$ & 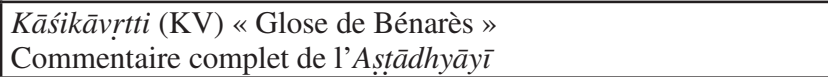 \\
\hline Kaiyața & $11^{\mathrm{e}} \mathrm{s} . ?$ & $\begin{array}{l}\text { Mahābhāsyapradīpa }(\mathrm{P}) \text { «Élucidation du grand commentaire » } \\
\text { Commentaire complet du Mahābhāsya }\end{array}$ \\
\hline Puruṣottama & $\begin{array}{l}\text { fin } 11^{\mathrm{e}} \\
\text { début } \\
12^{\mathrm{e}} \mathrm{s}\end{array}$ & $\begin{array}{l}\text { Prākrtānuśāsana «Instruction du prakrit » } \\
\text { Ensemble des règles phonétiques et morphologiques permettant } \\
\text { de former les mots de la māhārāstrīì à partir de la norme sanskrite, } \\
\text { ainsi que les règles concernant les autres dialectes s'écartant de la } \\
\text { norme prakrite }\end{array}$ \\
\hline Nāgeśa & $\begin{array}{l}\text { fin } 17^{\mathrm{e}} \\
\text { début } \\
18^{\mathrm{e}} \mathrm{s} \text {. }\end{array}$ & 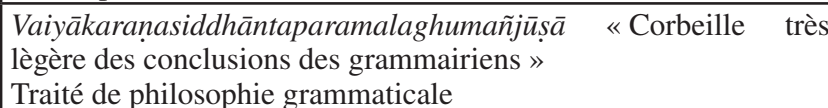 \\
\hline
\end{tabular}

\section{RÉFÉRENCES}

\section{Sources primaires}

Bhartṛharis Vākyapadīya. Die Mūlakārikas nach den Handschriften herausgegeben und mit einem Pāda-Index versehen von Wilhelm Rau, Wiesbaden, Franz Steiner (Abhandlung für die Kunde des Morgenlandes, 42.4), 1977.

La grammaire de Pānini. Texte sanskrit, traduction française avec extraits des commentaires par Louis Renou, Paris, École française d'Extrême-Orient, 2 vol., 1966.

Kāsikika of Pt. Vāmana and Jayāditya. Edited with the Prakāśa Hindi Commentary and Introduction by Śrī Nārāyaṇa Miśra, Varanasi, Chaukhambha Sanskrit Sansthan, 2 vol., 2000 ( $8^{\mathrm{e}}$ édition).

The Nātyaśāstra of Bharata Muni. Edited by Pandit Śivadatta and Kāśināth Pāṇurang Parab, Bombay, The Nirnaya Sagara Press, 1894.

The Nighantu and the Nirukta - The Oldest Indian Treatise on Etymology, Philology and Semantics. Edited by Lakshman Sarup, Delhi, Motilal Banarsidass, 1998 (réimpression de la $1^{\text {re }}$ édition indienne).

Patañjali's Vyākarana Mahābhāṣyam with Kaiyața's Pradīpa and Nāgojibhațta's Uddyota. Notes by A. G. Shastri, edited by Dr. Bal Shastri, Delhi, Pratibha Prakashan, 6 vol., 2001 ( $1^{\text {re }}$ édition : 1938).

Le Prākrtānuśásana de Purusottama. Par Luigia Nitti-Dolci, Paris, Cahiers de la Société asiatique VI, 1938.

The Prākrita Prakāśa of Vararuci. With the Sañjīvanī-Subodhinī-Manoramā and Prākrtamañjarī Commentaries and Hindi Translation, Ācāryaśrī Baladeva Upādhyāya (éd.), foreword by Dr. Mandan Mishra, Varanasi, Sarasvatībhavana Granthamālā vol. 102, 1996.

Śatapatha Brāhmana of the White Yayurveda, with the commentary of Sāyana Ācārya. 
Edited by Ācārya Satya Vrata Sāmasramī, Calcutta, The Asiatic Society (Bibliotheca Indica), vol. 3, kānda 3, 1905.

Vaiyākaranasiddhāntaparamalaghumañjūșā of Śri Nāgeśa Bhațta, with the commentary Jyotsnā. Edited by Pt. Kālikāprasād Shukla, Baroda, University of Baroda, 1961.

The Vyākarana-Mahābhāsya of Patañjali - Edited by F. Kielhorn. Revised and Furnished with Additional Readings, References, and Select Critical Notes by Kashinath Vasudev Abhyankar, Poona, Bhandarkar Oriental Research Institute (Bombay Sanskrit and Prakrit Series 18-20, 21-22 \& 26, 28-30), (1 $1^{\mathrm{e} e ́ d i t i o n ~(3 ~ v o l .) ~: ~ B o m b a y, ~ T h e ~ D e p a r t m e n t ~}$ of Public Instruction (1880-1885), vol. 1 : (troisième édition) 1962, vol. $2:$ (3 édition) 1965, vol. 3 : (3 édition) 1972.

\section{Sources secondaires}

Aklujkar, Ashok (1996). « The Early History of Sanskrit as Supreme Language », Houben, Jan E. M. (éd.), Ideology and Status of Sanskrit : Contributions to the History of the Sanskrit Language, Leiden, Brill, 59-85.

Balbir, Nalini (2000a). «Vararuci », Colombat, Bernard (éd.), Corpus représentatif des grammaires et des traditions linguistiques II, Histoire Épistémologie Langage Hors-Série 3, 267-269.

Balbir, Nalini (2000b). «Purusottama ou Purusottamadeva », Colombat, Bernard (éd.), Corpus représentatif des grammaires et des traditions linguistiques II, Histoire Épistémologie Langage Hors-Série 3, 273-275.

Bansat-Boudon, Lyne (1992). Poétique du théâtre indien. Lectures du Nātyaśāstra, Paris, Publications de l'École française d'Extrême-Orient.

Biardeau, Madeleine (1964). Théorie de la connaissance et philosophie de la parole dans le brahmanisme classique, Paris - La Haye, Mouton \& Co.

Bloch, Jules (1934). L'indo-aryen - Du Veda aux temps modernes, Paris, Librairie d'Amérique et d'Orient - Adrien Maisonneuve.

Bordier, Jean-Pierre (2002). Langues, codes et conventions de l'ancien théâtre-Actes de la troisième rencontre sur l'ancien théâtre européen (Tours, Centre d'études supérieures de la Renaissance, 23-24 septembre 1999), articles réunis et présentés par Bordier, Jean-Pierre, Paris, Honoré Champion Éditeur.

Bubeník, Vít (1998). A historical syntax of late middle indo-aryan (apabhramśa), Amsterdam - Philadelphia, John Benjamins Publishing Company.

Canut, Cécile (2001). "À la frontière des langues - Figures de démarcation », Cahiers d'études africaines 163-164, 443-464.

Cardona, George (1976). Pānini - A survey of research, Delhi, Motilal Banarsidass.

Cardona, George (1997). Pānini - His Work and its Traditions, Delhi, Motilal Banarsidass. [Seconde édition, revue et élargie, $1^{\mathrm{e}}$ édition : 1988]

Deshpande, Madhav M. (1978). «Pāninian Grammarians on Dialectal Variation », The Adyar Library Bulletin 42, 61-114.

Deshpande, Madhav M. (1993). «Historical Change and the Theology of Eternal (nitya) Sanskrit », Sanskrit and Prakrits - Sociolinguistic Issues, Delhi, Motilal Banarsidass, 53-74.

Deshpande, Madhav M. (1998). «Evolution of the Notion of Authority (Prāmānya) in the Pāninian Tradition », Histoire Épistémologie Langage 20/1, 5-28.

Driem, George, van (2001). Languages of the Himalayas - An Ethnolinguistic Handbook of the Greater Himalayan Region, Leiden, Brill.

Ghosh, Manomohan (1934). « The Date of the Bharata-Nātyasáastra », Journal of the Department of Letters 25,1-52.

Grierson, George A. (1901). « Review of Pischel's Prakrit Grammar », The Indian Antiquary 30, 553-556.

Grierson, George A. (1918). « The Prakrit Vibhasas », Journal of the Royal Asiatic Society, 489-517.

Hinüber, Oskar, von (1985). «Pāli and Paiśācī as Variants of Buddhist Middle Indic », 
Bulletin d'études indiennes 3, 61-77.

Hock, Hans Henrich (1999). « Through a glass darkly: Modern "racial" interpretations vs. textual and general prehistoric evidence on ārya and dāsa/dasyu in Vedic society », Bronkhorst, Johannes and Deshpande, Madhav (ed.), Aryan and non-aryan in South Asia - Evidence, Interpretation and Ideology, Cambridge, Harvard Oriental Series, Opera Minora vol. 3, 145-174.

Hock, Hans Henrich et Pandharipande, Rajeshwari (1978). «Sanskrit in the Pre-Islamic Sociolinguistic Context of South Asia », International Journal of the Sociology of Language 16, 11-25.

Houben, Jan E. M (1996). « Sociolinguistic Attitudes Reflected in the Work of Bhartrhari and Some Later Grammarians », Houben, Jan E. M. (éd.), Ideology and Status of Sanskrit : Contributions to the History of the Sanskrit Language, Leiden, Brill, 157-193.

Kane, Pandurang Vaman (1961). History of Sanskrit Poetics, Delhi, Motilal Banarsidass.

Kahrs, Eivind (1992). « What is a tadbhava word? », Indo-Iranian Journal 35, 225-249.

Kiparsky, Paul (1979). Pānini as a Variationist, Poona, Centre of Advanced Study in Sanskrit.

Kuiper, Franciscus Bernardus Jacobus (1997). « The genesis of a linguistic area », Lubotsky, Alexander, Oort, Marianne S., Witzel, Michael (éd.), F.B.J. Kuiper-Selected writings on Indian linguistics and philology, Amsterdam, Rodopi, Leiden Studies in Indo-European 8, 78-91. [1 $1^{\text {re }}$ édition : 1965]

Limaye, Vishnu Prabhakar (1974). Critical studies on the Mahābhāsya, Hoshiarpur, Vishveshvaranand Vedic Research Institute.

Mayrhofer, Manfred (1956-1976). Kurzgefasstes etymologisches Wörterbuch des Altindischen, Heidelberg, Carl Winter. [I. Band, A-TH (1956), II. Band, D-M (1963), III. Band, Y-H (1976[1964]), IV. Band, Register (1980[1978])]

Nitti-Dolci, Luigia (1938). Les grammairiens prakrits, Paris, Librairie d'Amérique et d'Orient, Adrien Maisonneuve.

Ojihara, Yutaka et Renou, Louis (1960-1967). La Kāśikā-Vrtti (adhyāya I, pāda 1), Traduction et commentaires, Paris, École Française d'Extrême-Orient, 3 vol. [vol. 1 : 1960, vol. 2 : 1962, vol. $3: 1967]$

Parpola, Asko (1994). Deciphering the Indus script, Cambridge, Cambridge University Press.

Pinault, Georges-Jean (1989a). «Reflets dialectaux en védique ancien », Caillat, Colette (éd.), Dialectes dans les littératures indo-aryennes - Actes du Colloque International, 16-18 septembre 1986, Paris, Collège de France, Institut de civilisation indienne, 35-96.

Pinault, Georges-Jean (1989b). «Travaux à partir du corpus védique », Auroux, Sylvain (éd.), Histoire des idées linguistiques I, Liège, Pierre Mardaga Éditeur, 303-330.

Pischel, Richard (1900). Grammatik der Prakrit-Sprachen. Strassburg, Trübner.

Pollock, Sheldon (2007). The Language of the Gods in the World of Men-Sanskrit, Culture, and Power in Premodern India, Delhi, Permanent Black.

Renou, Louis (1956). Histoire de la langue sanskrite, Paris - Lyon, Éditions IAC.

Renou, Louis, Filliozat, Jean \& al. (1985). L'Inde classique - Manuel des études indiennes I, Paris, Librairie d'Amérique et d'Orient, Adrien Maisonneuve. [Réimpression de l'édition de 1947]

Subramanya Iyer, Koduvayura Anantarama (1964). «Bhartṛari on apabhramśa», Vishveshvaranand Indological Journal 2/2, 242-246.

Thieme, Paul (1935). Pānini and the Veda - Studies in the Early History of Linguistic Science in India, Allahabad, Globe Press.

Thieme, Paul (1937). «Pānini and the Rkprātiśākhya », Indian Historical Quarterly 13 329-343.

Thieme, Paul (1955). « Review of The Sanskrit Language by T. Burrow », Language 31/3, 428-448.

Witzel, Michael (1999). «Aryan and non-Aryan Names in Vedic India. Data for the 